\title{
Nanospheres and nanocapsules of amphiphilic copolymers constituted by methoxypolyethylene glycol cyanoacrylate and hexadecyl cyanoacrylate units
}

\author{
I. Valente ${ }^{1}$, L. J. del Valle ${ }^{2}$, M. T. Casas ${ }^{2}$, L. Franco $^{1}$, A. Rodríguez-Galán ${ }^{2}$, J. Puiggali ${ }^{*}$, \\ D. Marchisio ${ }^{1}$
}

${ }^{1}$ Dipartimento di Scienza Applicata e Tecnologia, Politecnico di Torino, Corso Duca degli Abruzzi 24, 10129 Torino, Italy ${ }^{2}$ Departament d'Enginyeria Química, Universitat Politècnica de Catalunya, Av. Diagonal 647, E-08028 Barcelona, Spain

\begin{abstract}
Nanospheres and nanocapsules of an amphiphilic copolymer having methylated polyethylene glycol and hexadecyl lateral groups were prepared by the solvent displacement method and using confined impinging jet mixers. Degradation, thermal properties and crystalline structure were investigated. Interestingly, pegylated chains hydrolyzed through ester bond cleavage, whereas the more hydrophobic hexadecyl ester groups were resistant to degradation in aqueous media. The copolymer crystallized from the melt, giving rise to spherulites with a negative birefringence and domains corresponding to crystallization of the different lateral groups. Size distribution and morphology of nanoparticles were mainly evaluated by electron microscopy. Nanocapsules were characterized by a stable membrane with a thickness close to $5 \mathrm{~nm}$ that allowed efficient encapsulation of a triglyceride oil. Triclosan was selected as an example of a hydrophobic drug to be loaded in both nanospheres and nanocapsules. The release behavior of these dosage forms was clearly different. Thus, the Burst effect was practically suppressed when using nanocapsules; in addition, these showed a sustained, controlled release over a greater time period. Antimicrobial activity of triclosan loaded nanospheres and nanocapsules was evaluated using Gram-negative and Gram-positive bacteria. The former were highly sensitive to the released triclosan whereas the latter strongly depended on the number of particles in the culture medium.
\end{abstract}

Keywords: biodegradable polymers, nanocapsules, drug release, antimicrobial activity, amphiphilic polymers

\section{Introduction}

Amphiphilic copolymers are interesting because of their ability to stabilize diverse interfaces in aqueous systems and, in particular, as drug delivery systems [1]. Amphiphilic compounds are characterized by the incorporation of polar and non-polar monomer units in a polymer chain. Their synthesis and preparation can be a difficult task, especially when controlling the ratio between both chemical units in the resulting polymer chain and the homogeneity of the final structure, is needed [2].
The utility of amphiphilic copolymers for delivery of therapeutic agents is due to their unique chemical composition, which allows micellization in aqueous solutions and formation of hydrophobic core regions. These serve as reservoirs for hydrophobic drugs that can be loaded with chemical, physical or electrostatic means, depending on the specific functionalities of the core-forming block and the solubilizate. Furthermore, surface modification of particulate carriers with hydrophilic and flexible chains prevents their recognition by macrophages and rapid

\footnotetext{
*Corresponding author, e-mail: Jordi.Puiggali@upc.edu

(C) BME-PT
} 
elimination from the bloodstream [3, 4]. Pluronics $^{\circledR}$, which is constituted by poly(ethylene oxide) and poly(propylene oxide) blocks [5], is currently a common studied system, although copolymers containing poly(L-amino acid) and polyester hydrophobic blocks are gaining interest [2].

Amphiphilic molecules of cyanoacrylate have also been widely studied as drug delivery systems. These compounds are now prepared mainly by radical copolymerization reactions in monophasic organic solvents like dimethyl formamide or butanone $[6,7]$, or in two-phase solvents where the monomers dissolve each in one of the two phases [8-10]. Another method of preparation of cyanoacrylate amphiphilic copolymers is based on the condensation reaction of methoxy polyethylene glycol cyanoacetate and hexadecyl cyanoacetate with formaldehyde in the presence of an organic solvent like methylamine [11]. These methoxy polyethylene glycol cyanoacrylate-co-hexadecyl cyanoacrylate copolymers, abbreviated as poly(MePEGCA-co-HDCA), can be prepared with modulated hydrophobicity/hydrohilicity by adjusting the MePEGCA/HDCA monomer ratio. Some previous works focused on the preparation of nanoparticles from poly(MePEGCA-co-HDCA) with a hydrophilic/hydrophobic monomer ratio of 1:4 by nanoprecipitation and emulsion/solvent evaporation methods $[11,12]$. Monomodal size distributions were obtained with characteristic mean particle sizes ranging between 98 and $199 \mathrm{~nm}$ and varying according to polymer concentration and the applied method. It was also demonstrated that nanoparticles have an adequate density of MePEG chains on their surface to provide enhanced stability in the blood compartment and ensure a long circulating characteristic [13, 14]. Nanoparticles of poly(alkyl cyanoacrylate) amphiphilic copolymers have recently been successfully used for other biomedical applications such as human brain endothelial cell imaging [15] and early Alzheimer's disease diagnosis and treatment [16].

Drug loaded nanoparticles of poly(MePEGCA-coHDCA) have also recently been prepared using a new methodology based on solvent displacement in confined impinging jet mixers (CIJM) [17]. These devices allow continuous production, facilitate scaling-up and guarantee controllable operating parameters and conditions to improve final nanoparticle properties [18]. The solvent (e.g. acetone or tetrahydrofurane) and the anti-solvent streams (e.g. water) are mixed under different flow conditions, defined by and labelled in terms of the anti-solvent/solvent flow rate ratio $(R)$ and the flow rate of the anti-solvent stream $\left(F_{\mathrm{W}}\right)$. Besides their usefulness to get nanospheres constituted by the polymer alone, CIJM allow also the preparation of nanocapsules containing an oily core. Miglyol 812, a triglyceride of caprylic and capric fatty acids, seems appropriate to provide an oil core where water-insoluble drugs remain dissolved, resulting in high payload and a low release profile $[19,20]$.

The present work is devoted to the improvement of the physico-chemical characterization of poly (MePEGCA-co-HDCA), paying attention to its morphology and crystalline structure and also to its degradability. Furthermore, morphological features of poly(MePEGCA-co-HDCA) nanospheres and nanocapsules prepared using CIJM are described, and finally the drug loading and release from these nanoparticles are compared by using triclosan as an example of hydrophobic drug.

\section{Experimental section}

\subsection{Materials}

Methoxy poly(ethylene glycol) (MePEG) with a number average molecular weight of $2000 \mathrm{~g} / \mathrm{mol}$ was purchased from Sigma-Aldrich (St. Louis, MO, USA). Poly(MePEGCA-co-HDCA) and poly(hexadecyl cyanoacrylate) (PHDCA) were synthesized as previously reported [11] being 3600 and $5100 \mathrm{~g} / \mathrm{mol}$, respectively, the number average molecular weights determined by gel permeation chromatography (GPC). Poly(MePEGCA-co-HDCA) was synthesized from a methoxypolyethylene glycol cyanoacetate to $n$-hexadecylcyanoacetate ratio of 1:4.

Triclosan for release experiments was purchased from Sigma Aldrich. A microbial culture was prepared with reagents and labware from Scharlab S.L. (Barcelona, Spain). The bacterial strains were Escherichia coli CECT 101 and Micrococcus luteus CECT 245 from the Spanish Collection of Type Culture (CECT, Valencia, Spain).

\subsection{Measurements}

Molecular weights were estimated by GPC using a liquid chromatograph (Shimadzu, model LC-8A, Shimadzu Corp., Tokyo, Japan) equipped with an Empower computer program (Waters Corporation, Massachusetts, USA). A PL HFIP gel column (Agilent Technologies Inc., CA, USA) and a refractive 
index detector (Shimadzu RID-10A, Shimadzu Corp., Tokyo, Japan) were employed. The polymer was dissolved and eluted in tetrahydrofurane at a flow rate of $0.5 \mathrm{~mL} / \mathrm{min}$ (injected volume $100 \mu \mathrm{L}$, sample concentration $1.5 \mathrm{mg} / \mathrm{mL})$. The average molecular weights were calculated using polystyrene standards.

${ }^{1} \mathrm{H}$ nuclear magnetic resonance spectroscopy $\left({ }^{1} \mathrm{H}\right.$ NMR $)$ spectra were acquired with a Bruker AMX-300 spectrometer (Bruker Corp., Bremen, Germany) operating at $300.1 \mathrm{MHz}$. Chemical shifts were calibrated using tetramethylsilane as an internal standard. Dried deuterated chloroform was used as the solvent.

Calorimetric data were obtained by differential scanning calorimetry (DSC) with a TA Instruments Q100 (New Castle, DE, USA) series equipped with a refrigerated cooling system (RCS) operating from -90 to $550^{\circ} \mathrm{C}$. Experiments were conducted under a flow of dry nitrogen with a sample weight of approximately $10 \mathrm{mg}$ while calibration was performed with indium. Heating and cooling runs were performed at rates of 20 and $10^{\circ} \mathrm{C} / \mathrm{min}$, respectively.

Wide angle $\mathrm{X}$-ray diffraction patterns were obtained using a PANalytical X'Pert diffractometer (Panalytical B.V. Almelo, Netherlands), $\mathrm{Cu} \mathrm{K} \mathrm{K}_{\alpha}$ radiation ( $\lambda=$ $0.1542 \mathrm{~nm}$ ) and a silicium monocrystal sample holder.

Spherulitic morphologies were studied using a Zeiss Axioskop 40 Pol light polarizing microscope (Carl Zeiss, Göttingen, Germany) equipped with a Linkam temperature control system configured by a THMS 600 heating and freezing stage connected to an LNP 94 liquid nitrogen cooling system. Micrographs were taken with a Zeiss AxiosCam MRC5 digital camera. A first-order red tint plate was employed to determine the sign of spherulite birefringence under crossed polarizers.

Spherulites for transmission electron microscopy (TEM) observations were grown from homogeneous melt-crystallized thin films placed between two cover glasses and prepared by evaporation of a dilute solution of the polymer in acetone. This experimental procedure made it possible to obtain films with an appropriate thickness for TEM observation.

The nanocapsule (NC) and nanospheres (NS) size distribution was determined by Dynamic Light Scattering (DLS, Zetasizer Nanoseries ZS90, Malvern Instrument Ltd., Malvern, UK), which measures accurately in the size range from $2 \mathrm{~nm}$ to $3 \mu \mathrm{m}$.
Morphological observations of nanoparticles and nanocapsules were carried out with a Philips TECNAI 10 transmission electron microscope (Philips Electron Optics, Eindhoven, Holland) at an accelerating voltage of $80 \mathrm{kV}$ and a Focus Ion Beam Zeiss Neon 40 instrument for scanning electron microscopy. Carbon coating was accomplished using a Mitec K950 Sputter Coater (Carl Zeiss, Göttingen, Germany) equipped with a film thickness monitor $k 150 \times$ (Quorum Technologies Ltd., West Sussex, UK).

Negative staining and direct observation were employed to image the particles by TEM. In some cases, shadowing with $\mathrm{Pt} / \mathrm{C}$ at an angle of $15^{\circ}$ was performed to observe surface details and obtain information on the thickness of the specimen. The negative staining solution was prepared by diluting a $4 \%$ phosphotungstic acid solution with ethanol $100 \%$ in a proportion 1 to 4 .

A UV-3600 spectrophotometer controlled by UVProbe v2.31 software (Shimadzu, Tokyo, Japan) was employed for triclosan load and release measurements.

\subsection{Preparation of unloaded and triclosan loaded nanospheres and nanocapsules of poly(MePEGCA-co-HDCA)}

Nanospheres and nanocapsules of poly(MePEGCAco-HDCA) containing triclosan were prepared by the solvent displacement method (or nanoprecipitation) [21] using a CIJM with two inlet jets of $1 \mathrm{~mm}$, one outlet jet of $2 \mathrm{~mm}$ and a mixing chamber of about $5 \mathrm{~mm}$. More geometrical details on this device can be found in previous work [18]. For the preparation of nanoparticles, $150 \mathrm{mg}$ of poly(MePEGCAco-HDCA) was dissolved in $25 \mathrm{~mL}$ of warm acetone (i.e. polymer concentration of $6 \mathrm{mg} / \mathrm{mL}$ ). To obtain triclosan loaded nanoparticles, $0.3 \%(\mathrm{w} / \mathrm{v})$ of triclosan was added to the acetone solution. This solution was injected into the mixer by an infusion pump at a flow rate of $120 \mathrm{~mL} / \mathrm{min}$ (KDS200, KD Scientific Inc., Holliston, MA, USA). Simultaneously, $25 \mathrm{~mL}$ of water was injected at the same rate through the second upstream inlet. $R$ and $F_{\mathrm{W}}$ processing parameters were consequently 1 and $120 \mathrm{~mL} / \mathrm{min}$, respectively. Particles, spontaneously formed in the mixer chamber, were evacuated by the outlet jet, and then quenched in $25 \mathrm{~mL}$ of water under magnetic stirring. Finally, the acetone was evaporated in 
a rotating device (30 minutes at room temperature) to obtain an aqueous suspension of nanospheres. Nanocapsules were prepared by the same procedure after adding $200 \mu \mathrm{L}$ of Miglyol 812 (i.e. oil concentration of $8 \mu \mathrm{L} / \mathrm{mL}$ ) to the initial organic solution of the copolymer in order to form the inner oily cavity. Nanocapsules had an oil to polymer mass ratio of 1.26 .

Nanosphere and nanocapsule suspensions were extensively dialyzed (Spectra/Por ${ }^{\circledR} 3500$ MWCO dialysis membrane, Spectrum, Huston, TX, USA) at $25^{\circ} \mathrm{C}$ for 4 hours against a Sörensen solution supplemented with $10 \%(\mathrm{v} / \mathrm{v})$ of ethanol in order to remove all unloaded triclosan. Aliquots of $200 \mu \mathrm{L}$ of the sample suspension before and after dialysis underwent ultra-sound treatments and extracted with $1 \mathrm{~mL}$ of $70 \%$ ethanol to quantify triclosan by ultraviolet (UV) analysis $(281 \mathrm{~nm})$. Calibration curves were obtained by plotting the absorbance measured at $281 \mathrm{~nm}$ against triclosan concentration.

Entrapment efficiency (EE) and drug loading (DL) were determined by Equations (1) and (2), respectively. Occurrence of degradation during dialysis was not considered for DL evaluation (i.e. Equation (2) was referred to the initial polymer mass).

$$
\begin{aligned}
& \mathrm{EE}[\%]=100 \cdot \frac{\text { Experimental drug loading }}{\text { Nominal drug loading }} \\
& \mathrm{DL}[\%]=100 \cdot \frac{\text { Mass of drug in particles }}{\text { Mass of polymefr in particles }}
\end{aligned}
$$

\subsection{Degradation experiments}

Degradation was performed using small polymer disks with a diameter of $1 \mathrm{~cm}$ and a thickness of $1 \mathrm{~mm}$ prepared by press molding of approximately $150 \mathrm{mg}$ of the material. The degradation medium consisted of $15 \mathrm{~mL}$ of milliQ water and two different degradation temperatures $\left(4\right.$ and $\left.18^{\circ} \mathrm{C}\right)$ were considered. Tablets were removed from the degradation medium at scheduled times, and were then well dried and weighed to calculate the weight loss. Some samples were also analyzed by ${ }^{1} \mathrm{H}$ NMR to evaluate the chemical degradation of the polymer.

\subsection{Release experiments}

$10 \mathrm{~mL}$ of the aqueous suspension containing triclosan loaded nanoparticles or nanocapsules $(3 \mathrm{mg} / \mathrm{mL})$ was confined in a dialysis bag which was introduced in a vessel equipped with magnetic stirring and containing $20 \mathrm{~mL}$ of the selected release medium (i.e. a mixture $(3 / 7 \mathrm{v} / \mathrm{v})$ of Sörensen medium $(\mathrm{pH}$ 7.4) and ethanol). Aliquots ( $1 \mathrm{~mL})$ were drawn at predetermined intervals to determine the amount of released triclosan by UV spectroscopy. The volume of the release medium was kept constant by addition of $1 \mathrm{~mL}$ of fresh medium after removal of each aliquot. All drug release tests were carried out using five replicates to control the homogeneity of the release and average the results. The triclosan remaining in the samples was determined again by UV analysis. In this case, aliquots $(200 \mu \mathrm{L})$ of the dialysis bag sample were sonicated and extracted with $1 \mathrm{~mL}$ of $70 \%$ ethanol to ensure complete dissolution of triclosan.

The release kinetics can be calculated from experimental results by several theoretical models, typically first-order [22, 23], Higuchi [24, 25] and their combination [26]. Thus, the Higuchi-equation (Equation (3)) and first-order (Equation (4)) models were used to describe the first $(0-60 \%)$ and second (40$100 \%)$ parts of the release, respectively:

$$
\begin{aligned}
& \frac{M_{\mathrm{t}}}{M_{0}}=k_{\mathrm{h}} t^{1 / 2}, \quad\left(0 \leq \frac{M_{\mathrm{t}}}{M_{0}} \leq 0.6\right) \\
& \ln \left(1-\frac{M_{\mathrm{t}}}{M_{0}}\right)=a-k_{\mathrm{l}} t, \quad\left(0.4 \leq \frac{M_{\mathrm{t}}}{M_{0}} \leq 1.0\right)
\end{aligned}
$$

where $k_{\mathrm{h}}$ is the Higuchi release constant, $k_{1}$ is the first-order release constant, $a$ takes into account the release in the first step, $M_{\mathrm{t}}$ is the percentage of drug released at time $t$, and $M_{0}$ is the drug equilibrium percentage (considered as the maximum drug percentage).

\subsection{Antibacterial activity}

The in-vitro antibacterial activity of triclosan loaded nanoparticles and nanocapsules was evaluated using the Gram-positive Micrococcus luteus (M.luteus) and Gram-negative Escherichia coli (E.coli) microorganisms.

Briefly, $7 \mathrm{~mL}$ of Luria-Bertani (LB) broth containing $10^{5} \mathrm{CFU} / \mathrm{mL}$ was mixed with $1 \mathrm{~mL}$ of the nanosphere or nanocapsule aqueous suspension in sterile tubes. These were inverted 4-6 times to ensure mixing and incubated for 24 and $48 \mathrm{~h}$ in a shaking incubator at $100 \mathrm{rpm}$ and a temperature of $37^{\circ} \mathrm{C}$. Cultures of LB broth without and with bacteria were 
performed as negative and maximum bacterial growth controls, respectively. Culture of pure LB broth was the negative control, and pure LB broth with nanoparticles or nanocapsules was also tested as turbidity blank.

Bacterial growth was determined by measuring turbidity at $600 \mathrm{~nm}$ by UV spectroscopy. The number of surviving bacteria was computed according to the relative growth rate (percentage), which was calculated from turbidity changes after 24 and $48 \mathrm{~h}$ of incubation. Activities were evaluated using six replicates for which the corresponding average value and standard deviation were determined. Two samples were considered statistically significantly different when ANOVA and $\chi^{2}$-test gave $p \leq 0.05$.

The antibacterial effect in a dose-response manner was also determined for triclosan-loaded nanocapsules by considering different dilutions of the sam- ple and evaluating the relative growth, as noted above. The dose-response effect was analyzed by a logistic model using OriginPro v8 software (Origin Microcal Corp., Norhampton, MA, USA).

\section{Results and discussion}

\subsection{Thermal characterization of poly(MePEGCA-co-HDCA)}

Poly(MePEGCA-co-HDCA) has a semicrystalline nature, as shown by the DSC scans in Figure 1a. Thus, the as-synthesized sample has a predominant peak at $53^{\circ} \mathrm{C}$ and a minor one close to $33-34^{\circ} \mathrm{C}$, which should correspond to the melting of crystalline domains of PEG and hexadecyl (HD) alkyl groups, respectively. The sample easily crystallized from the melt, giving rise to a complex exothermic peak where the crystallization of the two indicated domains could not be well differentiated. It is inter-


Figure 1. DSC scans performed on poly(MePEGCA-co-HDCA) (a), PHDCA (b) and PEG (c) samples. Scans from bottom to top correspond to the heating run of the as-synthesized and the commercial samples, the cooling run from the melt state, the heating run of a hot crystallized sample and the heating run of a sample quenched from the melt state (a). 
esting to note that the crystallinity of the as-synthesized sample, which came from evaporation of a dichloromethane solution, was slightly higher than that of the hot crystallized sample (i.e. the global melting enthalpy changed from 133 to $125 \mathrm{~J} / \mathrm{g}$ ). This increase was caused by the higher endothermic peak associated with the PEG domain $\left(\mathrm{ca} .53^{\circ} \mathrm{C}\right.$ ) that could overcome the more deficient arrangement of the HD domain, which led to a very low melting peak at $33.4^{\circ} \mathrm{C}$. This peak clearly increased after hot crystallization indicating an improved packing of HD chains. Some differences are also found in the melting peak associated with the PEG domain since a shoulder, which is indicative of a typical re-crystallization or lamellar thickening process, was observed for the as-synthesized sample whereas only a single peak was detected for the hot crystallized sample.

The DSC heating run of a quenched sample does not reveal significant changes and demonstrates that the sample crystallized easily even at the high cooling rates given by the equipment. Note that the glass transition temperature could not be well observed due to the high crystallinity of the sample but it should be close to $-63^{\circ} \mathrm{C}$, as reported for PEG of low molecular weight [27]. Two points are worth highlighting from the thermal analysis: a) The sample undergoes a partial fusion at around $34^{\circ} \mathrm{C}$, a temperature slightly lower than the human body temperature, at which the potential drug delivery systems should be applied; b) Despite the complexity of the sample, its crystallinity remains high at $37^{\circ} \mathrm{C}$, which is the temperature at which samples are meant to be used.

For the sake of completeness, Figures $1 \mathrm{~b}$ and $1 \mathrm{c}$ show representative DSC scans performed with PHDCA and PEG homopolymers, respectively. These traces clearly confirm the previous assignation given for the melting peaks of poly(MePEGCAco-HDCA) and the similar crystallization temperature of both samples. Results also demonstrated that poly(MePEGCA-co-HDCA) has an intermediate melting enthalpy between those of the crystals constituted by the two types of lateral chains. The enthalpies associated with each peak (e.g. 21 and $104 \mathrm{~J} / \mathrm{g}$ for the hot crystallized sample) fit reasonably well with the expected values (20 and $103 \mathrm{~J} / \mathrm{g}$ ) assuming a weight percentage of PEG close to $61 \%$. Finally, the complexity of the crystallization exothermic peak of PEG, which extends over an interval of approximately $15^{\circ} \mathrm{C}$, and the sharp appearance of the peak associated with the PHDCA homopolymer are worth noting. This also suggests a practically instantaneous primary crystallization that might result from a high nucleation density.

\subsection{Spherulitic morphologies of poly(MePEGCA-co-HDCA)}

Crystallization from the melt led to the formation of spherulites with a fibrillar/ringed texture corresponding to the crystallization of the PEG lateral chains, although domains constituted by HD units could also be envisaged. Figure 2a shows a typical crystallization performed at $4{ }^{\circ} \mathrm{C}$ (i.e. at a low degree of supercooling) where well developed spherulites were observed, together with zones with a different texture (indicated by arrows in Figure 2a) that seem to be constituted by smaller microcrystals. These zones melted when the sample was heated to the melting temperature associated with the PHDCA domains (Figure $2 b$ ) and re-crystallized, giving textures similar to those initially observed when the temperature was decreased to room temperature (Figure 2c). Experiments clearly highlighted the complex crystallization process of samples constituted by blocks able to crystallize independently (i.e. those constituted by the PEG and HD lateral groups) and furthermore with a similar crystallization temperature. Phase separation and crystalline morphology studies of block copolymers are currently receiving much attention [28-32], and even microstructures that can be formed from the melt, from solution and for both thin and bulk samples have been extensively reviewed [33, 34]. Ringed spherulites with an interspacing close to $9 \mu \mathrm{m}$ could also be detected in the specimens (Figure 2d) although at a lower ratio.

A fibrillar spherulitic texture was observed when crystallization was performed at lower temperatures (e.g. $-24^{\circ} \mathrm{C}$ ), as shown in Figure 2e. The nucleation density obviously increased with decreasing the crystallization temperature; consequently, smaller spherulites were found at the end of the crystallization process. In this way, an increase from 20 to 600 nuclei $/ \mathrm{mm}^{2}$ was determined when the temperature decreased from 4 to $-24^{\circ} \mathrm{C}$.

In all cases, a negative birefringence was characteristic of the PEG spherulites, whereas a more confusing sign was found for the domains constituted by the HD alkyl chains due to their smaller size. In 


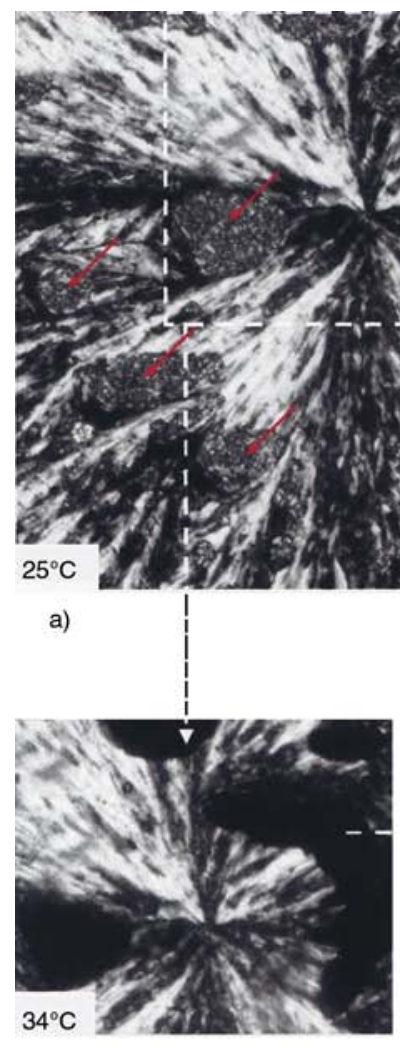

b)

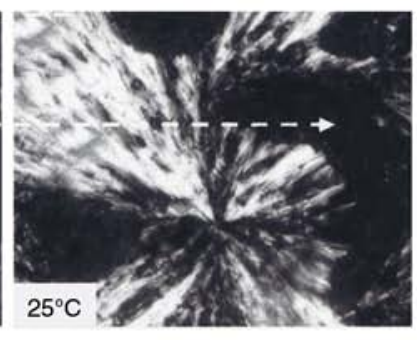

c)

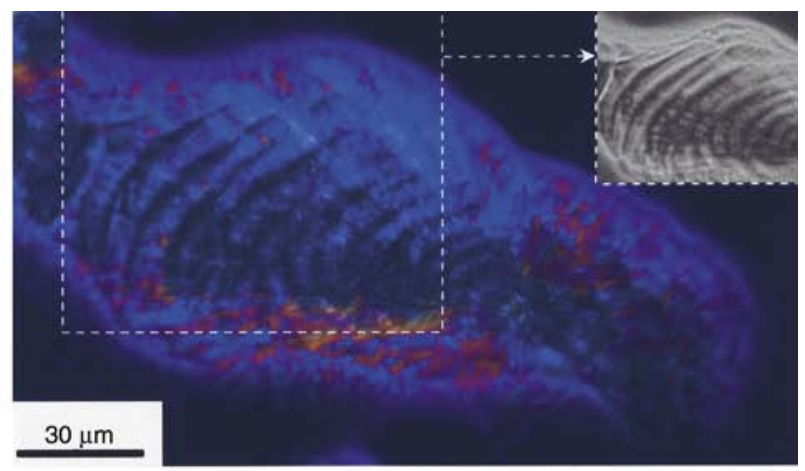

d)

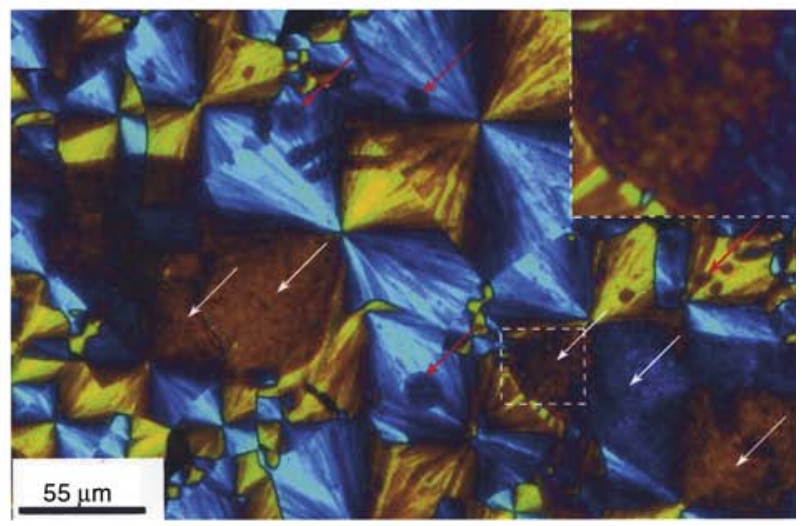

e)

Figure 2. Polarizing optical micrographs of poly(MePEGCA-co-HDCA) isothermally crystallized at $4^{\circ} \mathrm{C}(\mathrm{a}, \mathrm{b}, \mathrm{c}, \mathrm{d})$ and $24^{\circ} \mathrm{C}(\mathrm{e})$. Micrographs were taken at room temperature (a, d, e), at $34^{\circ} \mathrm{C}(\mathrm{b})$ and at room temperature after heating the crystallized sample to $34^{\circ} \mathrm{C}$ (c). A first-order red tint plate was used for micrographs (d) and (e). Inset of (d) shows a contrast phase image of a ringed spherulite. The inset of (e) shows a magnification of the dashed area where small and flat microcrystals can be envisaged. Arrows point to crystalline microdomains constituted by PHDCA.

fact, primary nucleation seemed to be much higher for these domains, giving rise to microcrystals. It should be pointed out that DSC data indicated that the hot crystallization of PHDCA proceeded rapidly, which was well justified by assuming a high primary nucleation. Optical micrographs revealed that the alkyl chain microcrystals had a flat appearance and usually appeared aggregated in such a way that the birefringence sign of the PEG spherulite was kept (see white and red arrows in Figure 2e). In some cases, these microcrystals led to the formation of spherulite arms with a speckled appearance (white arrows).

Phase separation was confirmed by electron diffraction since fibrillar textures and microcrystals gave rise to the typical $h k 0$ pattern of PEG and a well differentiated pattern with six reflections at $0.415 \mathrm{~nm}$, respectively. This pattern (Figure 3 ) suggests a pseudo-hexagonal packing of alkyl chains, which is consistent with the well known first-order transition undergone by long-chain paraffins at a few degrees below their melting point [35].
Figure 3. Electron diffraction patterns taken from fibrilar spherulites corresponding to HD domains 


\subsection{Crystalline structure of poly(MePEGCA-co-HDCA)}

X-ray powder diffraction patterns (Figure 4a) of poly(MePEGCA-co-HDCA) revealed the presence of reflections characteristic of polyethylene glycol, as well as additional peaks which should be assigned to a crystalline structure associated with the packing of the hexadecyl lateral groups. The structure of polyethylene glycol is defined by a $\mathrm{P} 21 / a$ space group and a unit cell with parameters $a=0.805 \mathrm{~nm}, b=$ $1.304 \mathrm{~nm}, c$ (fiber axis) $=1.948 \mathrm{~nm}$ and $\beta=125.4^{\circ}$ that contains four $7 / 2$ helices based on TTG sequences [36] (Figure 4b). The corresponding Xray diffraction pattern (Figure $4 \mathrm{c}$ ) was characterized by strong peaks at $0.462 \mathrm{~nm}$ (120 reflection) and $0.386-0.277 \mathrm{~nm}(112,032,132$ and 212 reflections) and weak peaks at 0.603 and $0.586 \mathrm{~nm}$, which are indexed as the 021 and 110 reflections.

All characteristic reflections of PEG, together with peaks at 2.988, 1.494 and $0.416 \mathrm{~nm}$ of remarkable intensity, can be well observed in the X-ray diffraction profile of poly(MePEGCA-co-HDCA). These

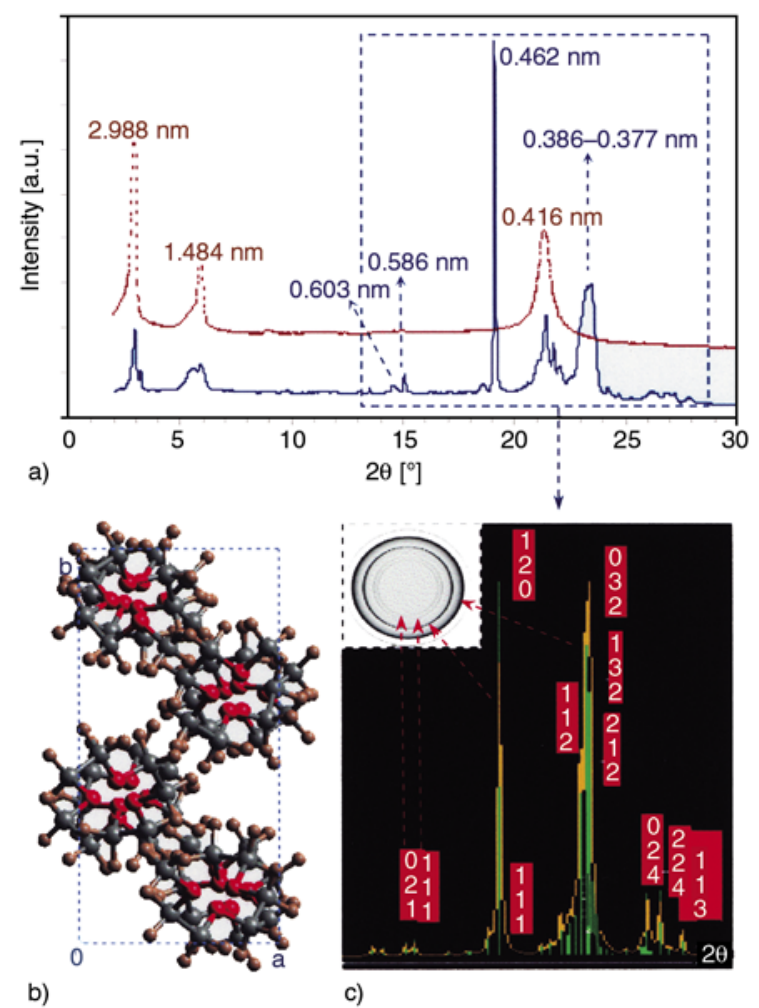

Figure 4. (a) Powder X-ray diffraction profiles of poly (MePEGCA-co-HDCA) (blue) and PHDCA (brown) samples. (b) Projection down the chain axis of the PEG structure showing the packing arrangement of the four $7 / 2$ helices. (c) Simulated powder X-ray diffraction profile of PEG and corresponding diffraction pattern (inset).

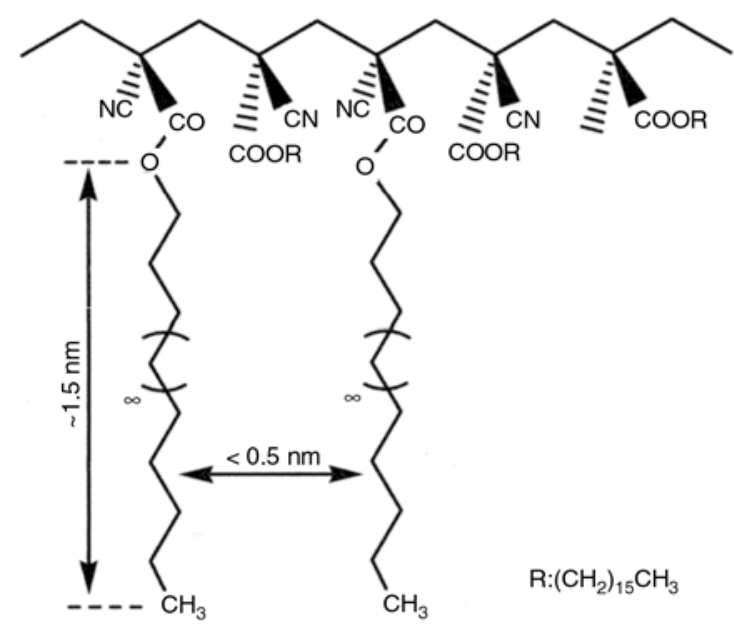

Figure 5. Schematic representation showing geometrical features concerning hexadecyl lateral groups of poly(MePEGCA-co-HDCA)

peaks, which are also detected in the diffractogram of PHDCA, suggest the presence of a hexagonal unit cell with parameters $a=0.479 \mathrm{~nm}, b=0.479 \mathrm{~nm}$, $c$ (fiber axis) $=2.988 \mathrm{~nm}$. Note that the strong peak at $0.416 \mathrm{~nm}$ corresponds to the 100,010 and 110 reflections, which were also observed in the electron diffraction patterns of HDCA spherulitic domains, and that are typical of a hexagonal packing of polymethylene segments. The higher spacing peaks can be indexed as the 001 and 002 reflections of a cell which $\mathrm{c}$ axis parameter corresponds to two lateral groups since the dimension of the alkyl side group becomes close to $1.5 \mathrm{~nm}$ (i.e. the 002 spacing) if an extended zig-zag conformation is assumed (Figure 5). It must also be pointed out that a syndiotactic disposition of the hexadecyl lateral groups is preferred to avoid high steric hindrances between the polymethylene sequences of neighboring chain units (i.e. a value close to $0.25 \mathrm{~nm}$ should be expected for an isotactic configuration).

\subsection{Hydrolitic degradation of poly(MePEGCA-co-HDCA)}

Degradation of poly(MePEGCA-co-HDCA) in distilled water at temperatures of 18 and $4^{\circ} \mathrm{C}$ occurred over a maximum period of $8 \mathrm{~h}$, followed by a slow process that extended over approximately $48 \mathrm{~h}$. After that, the weight of the copolymer samples remained practically constant, at least over an exposure time of $300 \mathrm{~h}$. The weight loss profiles at the two assayed temperatures were similar, although a slightly higher mass loss was detected at $18^{\circ} \mathrm{C}$ (Figure 6). Thus, weight losses of 63 and $67 \%$ were 

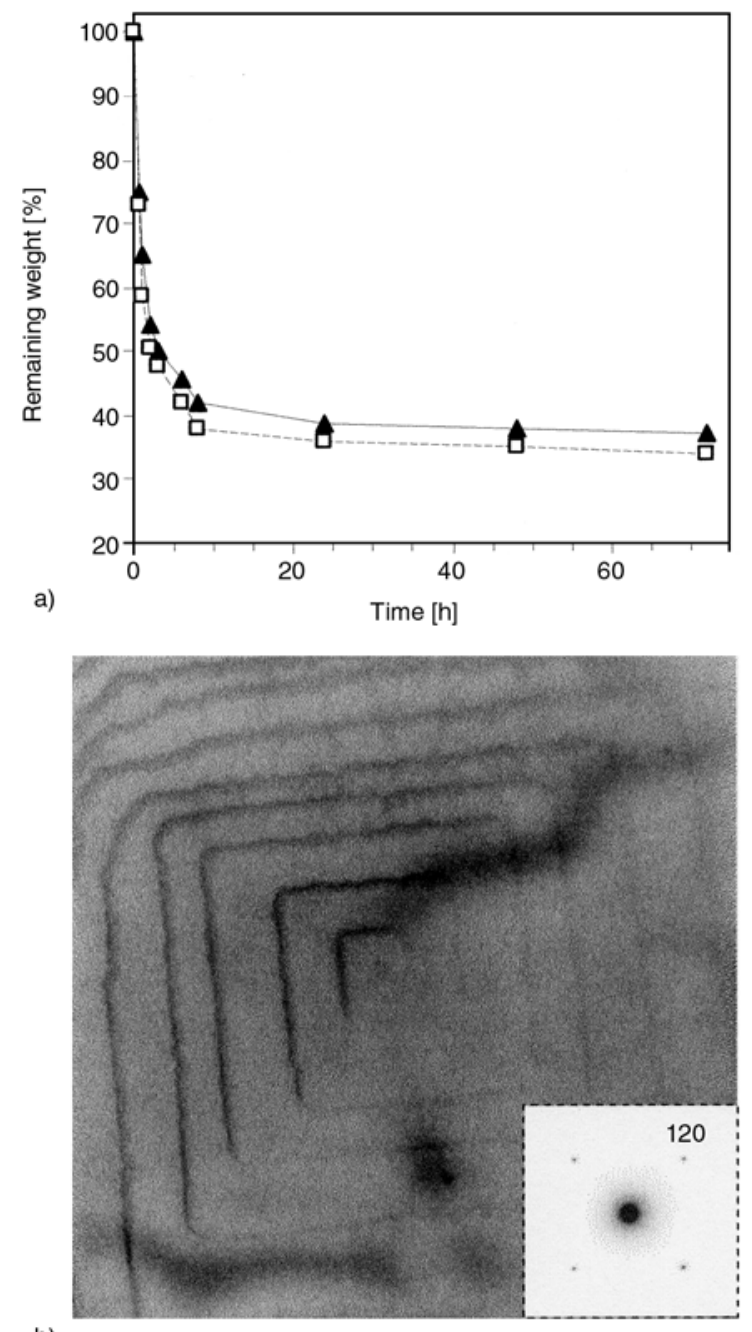

b)

Figure 6. (a) Plot of the remaining weight of a poly (MePEGCA-co-HDCA) disk sample versus exposure time in distilled water at $18(\square)$ and $5^{\circ} \mathrm{C}(\boldsymbol{\Delta})$. (b) Electron micrograph of PEG lamellar crystals recovered from the release medium. Inset shows the corresponding electron diffraction pattern.

determined at the end of the test performed at 4 and $18^{\circ} \mathrm{C}$, respectively. Enhanced solubility at the higher temperature is the probable cause of the slight differences in the remaining weight.

As already mentioned the copolymer was synthesized from a hydrophilic-to-hydrophobic monomer ratio of 1:4. However, this ratio should represent an 'average' value of the units incorporated to the polymer chain, and although the synthesis protocol is quite robust, it is likely that a distribution of hydrophilic-to-hydrophobic unit ratios exists in the final copolymer molecules. Thus, a significant weight loss should be expected from the presence of soluble fractions constituted by molecules with ratios of 1:2 or higher. The remaining part of the weight loss should be attributed to the hydrolysis of the ester groups of the hydrophilic lateral pegylated chains. Figures $7 \mathrm{a}$ and $7 \mathrm{~b}$ compare the ${ }^{1} \mathrm{H}$ NMR spectra of the as-synthesized sample and that degraded up to a weight loss of $67 \%$. The initial sample was characterized by an average molar ratio close to $1: 4$ between pegylated and hexadecyl (HD) lateral chains, as can be deduced from the areas of the signals at 3.37 and $0.86 \mathrm{ppm}$ assigned to the methyl groups belonging to the two ester moieties [11]. Methylene groups of the main chain and the lateral hexadecyl and PEG groups also appeared well differentiated at 2.37, 1.25 and $3.64 \mathrm{ppm}$, respectively (Figure 7a).

Spectra of samples exposed to water clearly show how the PEG signals at 3.64 and 3.37 ppm practically disappeared while HD signals at 1.25 and $0.86 \mathrm{ppm}$ remained with a remarkable intensity. In fact the hydrophilic-to-hydrophobic unit ratio decreases up to 1:110. Thus, the degradation process could be justified by an ester group cleavage involving mainly the pegylated chains.

According to the composition determined from NMR spectra, the MePEG lateral groups represented $55 \mathrm{wt} \%$ of the copolymer, which was slightly lower than the experimental weight loss. Thus, a percentage of the initial sample (i.e. chains with a high hydrophilic content) should be dissolved during exposure to the aqueous medium. In order to estimate this fraction, the as-synthesized sample was extracted with water in a time period during which degradation was practically negligible (i.e. less than $5 \%$ for $30 \mathrm{~min}$ of exposure). The extracted fraction corresponded to $10 \mathrm{wt} \%$ of the sample and its NMR spectrum (Figure 7c) indicated a 1:1.4 ratio between MePEG and HD lateral chains that was in agreement with the ratio expected for soluble molecules.

The preferential hydrolysis of pegylated chains was also corroborated by the ${ }^{1} \mathrm{H}$ NMR spectra (not shown) of the residue extracted from the hydrolytic degradation medium after $72 \mathrm{~h}$ of exposure, which indicated a PEG/HD ratio higher than 15:1. Furthermore, lamellar crystals with the characteristic morphology of polyethylene glycol lamellae [37, 38] were recovered after evaporation of the degradation medium. Specifically, Figure $6 \mathrm{~b}$ shows typical square lamellae of PEG and the corresponding electron diffraction pattern with intense 120 reflections at $0.462 \mathrm{~nm}$. 


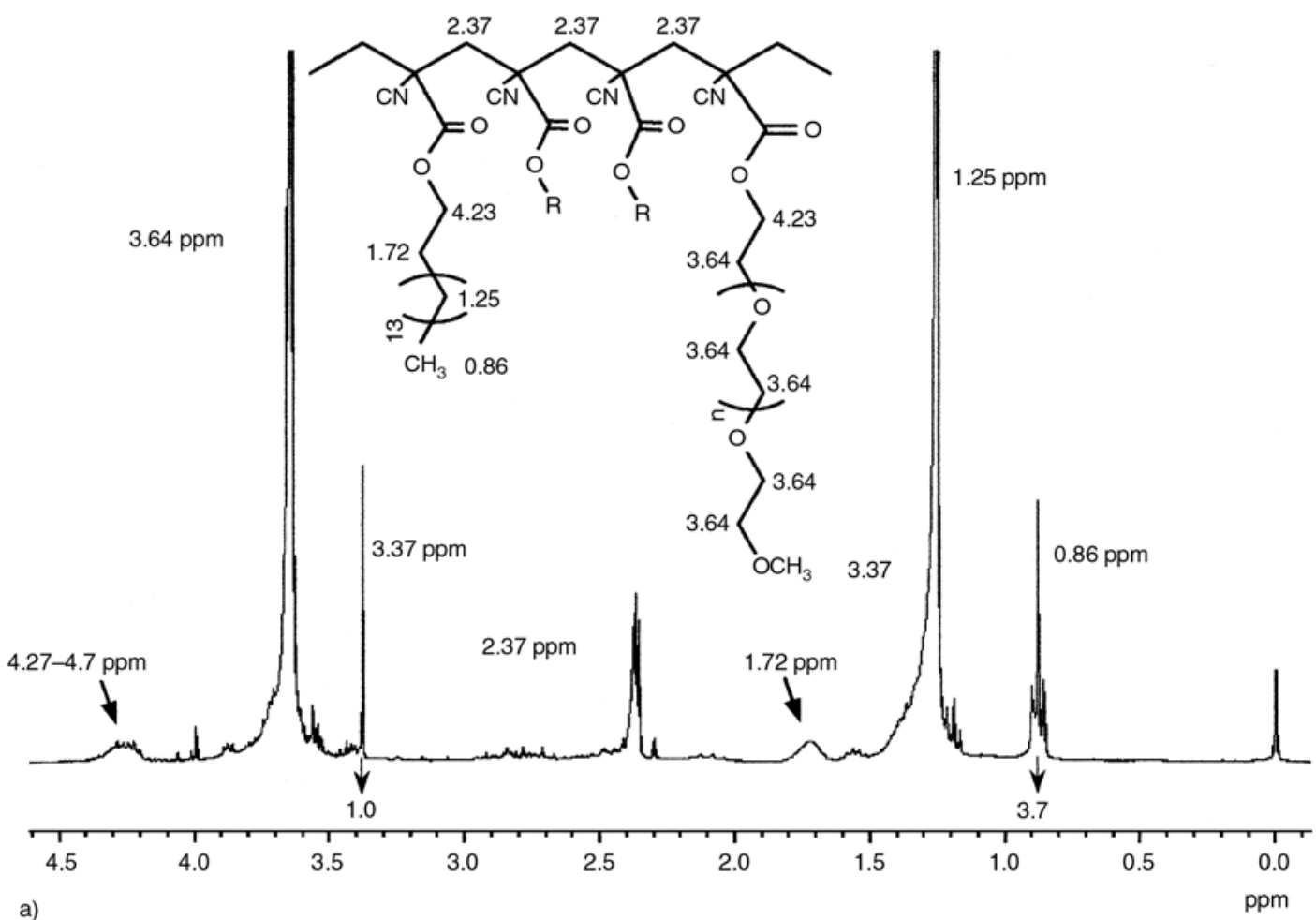

a)

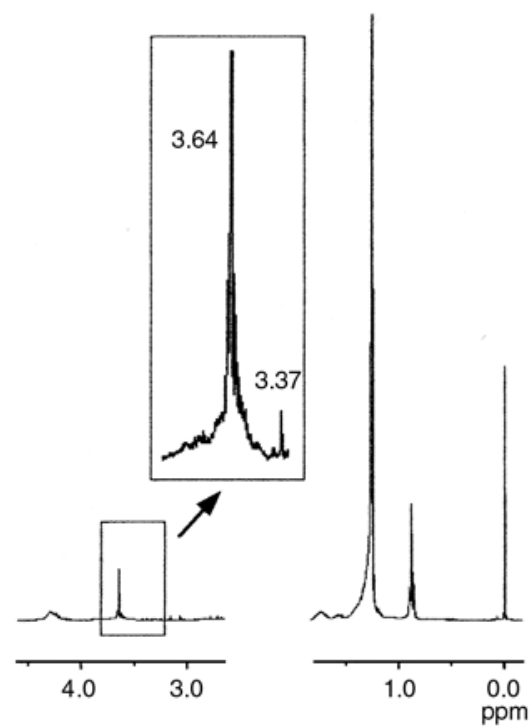

b)

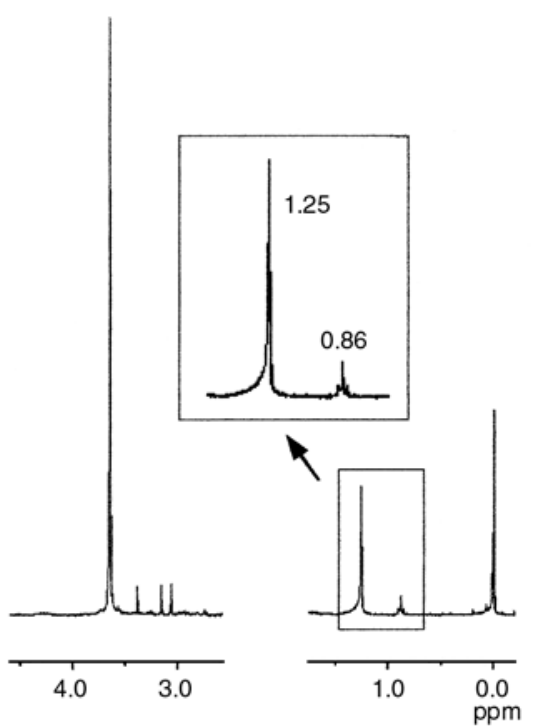

Figure 7. (a) ${ }^{1} \mathrm{H}$ NMR spectra of the as-synthesized poly(MePEGCA-co-HDCA) sample and chemical scheme of the assignment of signals (inset). (b) ${ }^{1} \mathrm{H}$ NMR spectra of a poly(MePEGCA-co-HDCA) sample after $72 \mathrm{~h}$ of exposure to water at $18^{\circ} \mathrm{C}$. (c) ${ }^{1} \mathrm{H}$ NMR spectra of the extracted fraction of a poly(MePEGCA-co-HDCA) sample after $30 \mathrm{~min}$ of exposure to water at $18^{\circ} \mathrm{C}$.

In conclusion, ${ }^{1} \mathrm{H}$ NMR spectra pointed out that the sample weight loss occurred through ester bond cleavage of the hydrophilic pegylated chains and by solubilisation of a small fraction corresponding to the more hydrophilic copolymer fraction. It is interesting to note that the ester bond of the hydrophobic moiety was not highly susceptible to hydrolysis probably due to poor exposure to the degradation medium. These results are in full agreement with preliminary studies on fetal calf serum which indicate that the hexadecyl homopolymer did not degrade during the first $3 \mathrm{~h}$ of incubation [12].

Finally, it is also interesting to note that degradation of pegylated chains can lead to the formation of anionic carboxylate groups, which still provide a hydrophilic character to the polymer. 


\subsection{Morphology of nanospheres and nanocapsules}

Particle size and size distribution are the most important characteristics of nanoparticle systems since they influence their biological fate, toxicity, targeting ability and even drug loading, drug release and stability. Many studies have demonstrated that nanoparticles of sub-micron size have a number of advantages over micro-particles, for example as a drug delivery system because of their small size and relative mobility. These properties can improve intracellular uptake and increase availability to a wider range of biological targets [39].

DLS analysis revealed a size difference between the triclosan loaded nanoparticles and nanocapsules. Thus, unimodal size distributions with mean diameters of 160 $600 \mathrm{~nm}$ (Figure 8a) and 230 $260 \mathrm{~nm}$ (Figure $8 \mathrm{~b}$ ), respectively, were found.

Scanning electron micrographs (Figure 8c) showed that nanoparticles were mostly spherical and had a rather variable diameter, in agreement with DLS results. Particles were generally found isolated although some aggregates could also be observed.

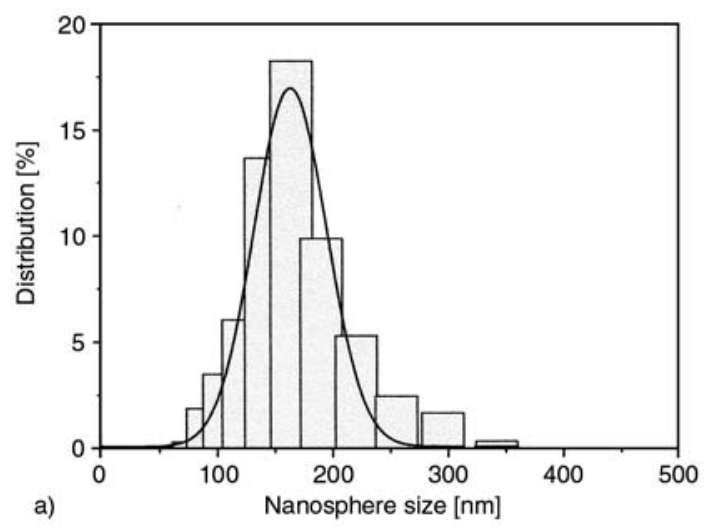

TEM micrographs taken at a higher magnification showed that particles, directly deposited on the carbon grid, were constituted by a porous dense polymer matrix (Figure 9a). Furthermore, these nanoparticles often appeared slightly deformed, their rounded appearance being lost, as clearly seen in the negative stained samples (Figure 9b). Nanoparticles were also shadowed with $\mathrm{Pt} / \mathrm{C}$ to determine their thickness and confirm their spherical morphology. Thus, the maximum and minimum shadows in the micrograph in Figure 10 correspond to thicknesses of 230 and $115 \mathrm{~nm}$, which are again in agreement with the particle size distribution determined by DLS measurements.

Medium magnification TEM images of nanocapsules (Figures 11a and 11c) showed their morphology and confirmed the underlying size distribution. This distribution extended over the 110-290 nm range, which covers smaller diameters than those deduced from DLS analysis. However, the predominant nanocapsule size (ca. $200 \mathrm{~nm}$ ) was close to the average value previously determined. Direct and negative stained images showed that isolated

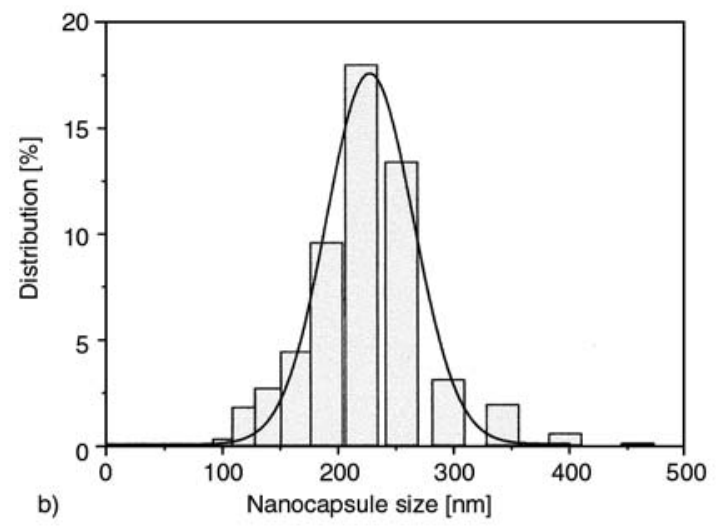

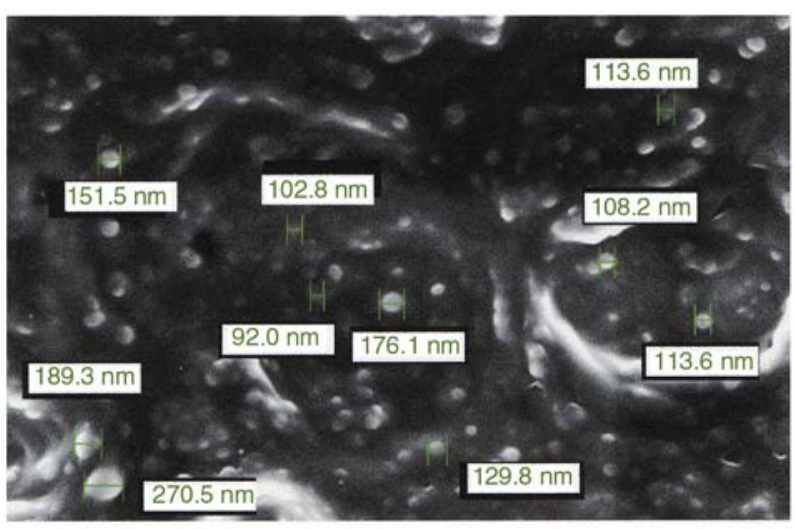

c)

Figure 8. Size distribution of triclosan loaded nanospheres (a) and nanocapsules (b) of poly (MePEGCA-co-HDCA). Samples were prepared with an initial copolymer concentration of $6 \mathrm{mg} / \mathrm{mL}$ with $R=1$ and $F R_{\mathrm{W}}=120 \mathrm{~mL} / \mathrm{min}$. For nanocapsules an oil concentration of $8 \mu \mathrm{L} / \mathrm{mL}$ was used. (c) Scanning electron micrograph of poly(MePEGCAco-HDCA) nanospheres prepared as above indicated. 


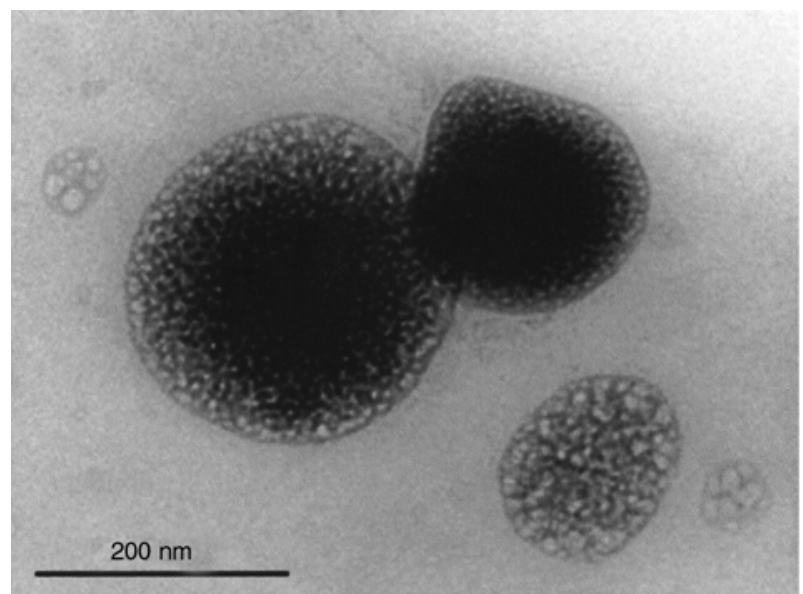

a)

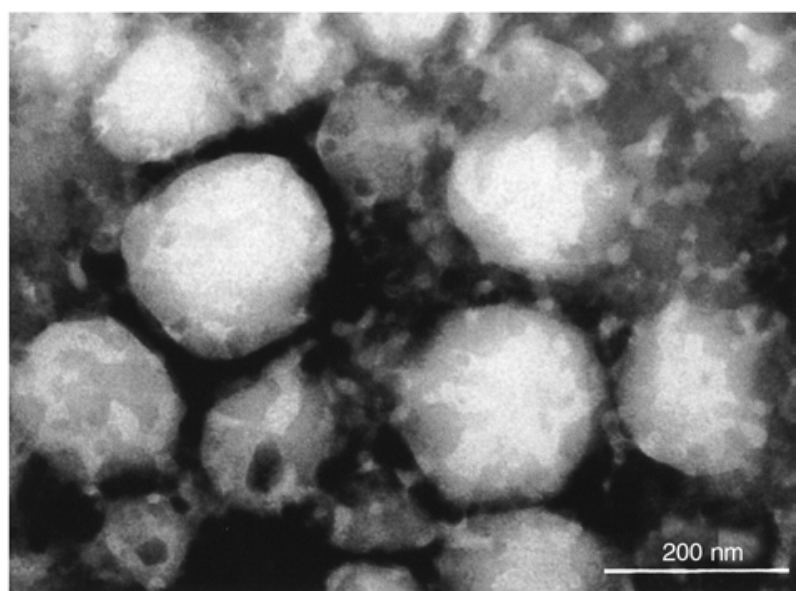

b)

Figure 9. Positive (a) and negative (b) TEM micrographs taken at different magnifications of poly(MePEGCA-co-HDCA) nanospheres. Samples were prepared with $R=1$ and $F R_{\mathrm{W}}=120 \mathrm{~mL} / \mathrm{min}$ and an initial copolymer concentration of $6 \mathrm{mg} / \mathrm{mL}$, respectively.

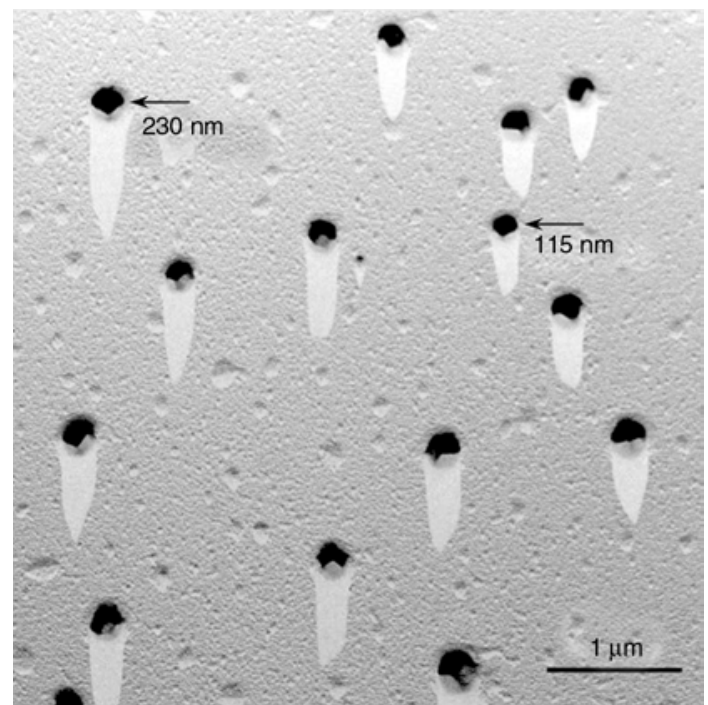

Figure 10. TEM micrograph of $\mathrm{Pt} / \mathrm{C}$ shadowed poly (MePEGCA-co-HDCA) nanospheres

nanocapsules were practically spherical but easily deformed when they were aggregated. Thus, the adjacent nanocapsule membranes became parallel and planar (see arrows in the negative stained sample of Figure 11c), showing that the spherical shape is actually not rigid. Both the liquid content and the reduced stiffness of the polymer membrane should be taken into account. The inset of Figure 11c contains the two wall membranes with a probable thickness of approximately $5 \mathrm{~nm}$, since it can be considered that the negativation salt had not penetrated in the nanocapsules or between adjacent membranes. Specimens obtained by direct deposition are sensitive to the large intensity of the electron beam. For this reason, some nanocapsules broke down during observation and released their liquid content. Note in Figure 11b some dense particles corresponding to the polymer membrane on the edges of the oil released by the breaking down of the nanocapsule. Figures $12 \mathrm{a}$ and $12 \mathrm{~b}$ reproduce bright field images of nanocapsules directly deposited onto the copper grid where the membranes can be clearly discerned in the projection contrast and a wall thickness close to $5 \mathrm{~nm}$ measured. Most nanocapsules were surrounded by a somewhat bright halo, which could be attributable to the oil released by the breaking down of the nanocapsule during observation.

The polymer matrix should be amorphous, but it is not discarded that side groups could crystallize. Moreover, some degraded pegylated chains and even some aggregated polymer molecules could be encapsulated, accounting for the dark zones found in nanocapsules (Figures 12a-12c). Pt/C shadowed samples (Figure 10d) revealed that nanocapsules usually collapse, giving rise to almost flat, rounded morphologies (note the shadow in Figure 12d, which suggests a small thickness). Flat morphologies were again a consequence of the oil release and the spongy structure of the polymer filled with liquid. It should be pointed out that shadowed samples allowed the detection of regular crystals inside the nanocapsule, as deduced from the surface details in Figure 12d. Specifically, aggregates of square crystals similar to those characteristic of PEG could be clearly envisaged. 


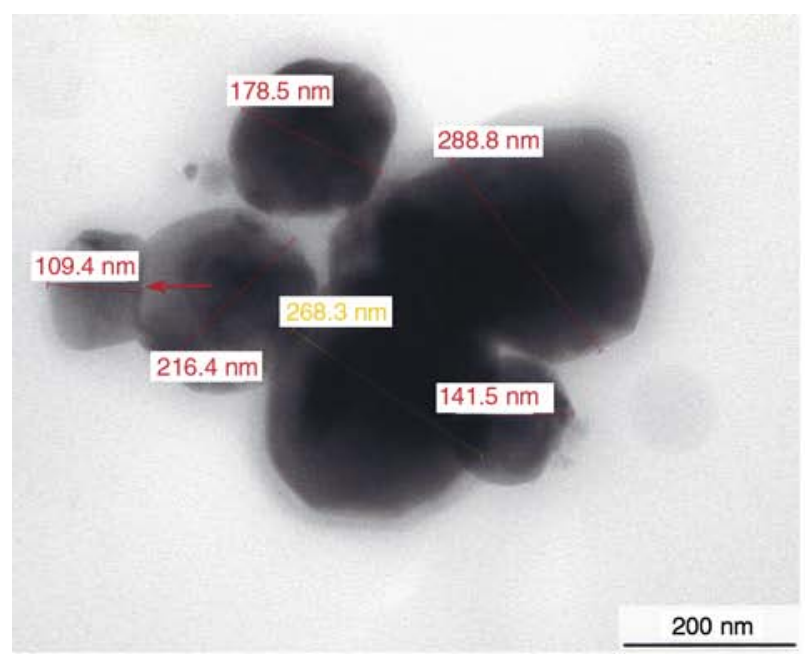

a)

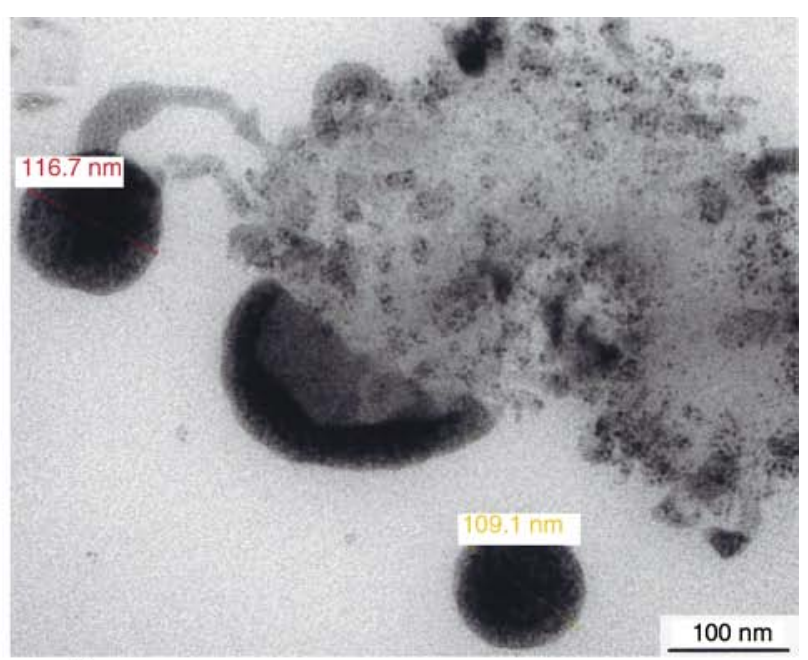

b)

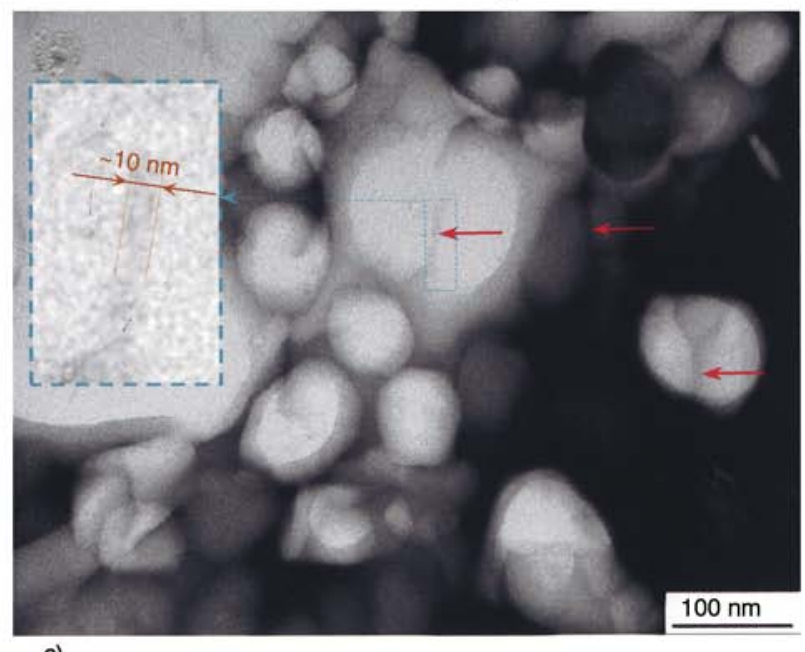

c)

Figure 11. Positive (a, b) and negative (c) TEM micrographs taken at different magnifications of poly(MePEGCA-coHDCA) nanocapsules containing Miglyol 812. Samples were prepared with $R=1$ and $F R_{\mathrm{W}}=120 \mathrm{~mL} / \mathrm{min}$ and an initial copolymer and oil concentration of 6 and $8 \mu \mathrm{L} / \mathrm{mL}$, respectively. Red arrows indicate close contact between nanocapsules that led to deformation. Inset of (c) shows a high magnification of the area of contact between two nanocapsules.

\subsection{Release of triclosan from nanospheres and nanocapsules}

Encapsulation efficiency (EE) was high and similar for both nanospheres and nanocapsules since values of 92 and $91 \%$ were respectively determined. It is clear that the polymer and the preparation method were effective in encapsulating high doses of triclosan. Drug loading (DL) was consequently relatively high and again similar for both systems (43 and $44 \%$ for nanospheres and nanocapsules, respectively). The amount of drug entrapped determines the performance of the drug delivery system as it influences the rate and extent of drug release from the system. Both DL and EE depend on physicochemical properties and the interactions between the drug, the carrier matrix, the encapsulated oil and the surrounding medium.
Triclosan release has been studied in Sörensen medium containing 70 volume percentage of ethanol in order to increase the hydrophobicity of the medium and avoid reaching an equilibrium condition. The studied mixture had previously been found to give similar triclosan release results in more expensive serum-based media [40].

Release of triclosan from nanoparticles and nanocapsules is clearly different, as compared in Figure 13a. Thus, the profile of nanospheres showed an initial Burst effect up to a percentage close to $35 \%$ which was mainly caused by the drug deposited on or close to the particle surface, followed by a slower, gradual release indicative of an effective hydrophobic interaction between the drug and the hydrophobic hexadecyl groups. On the contrary, the Burst effect was not significant when using nano- 


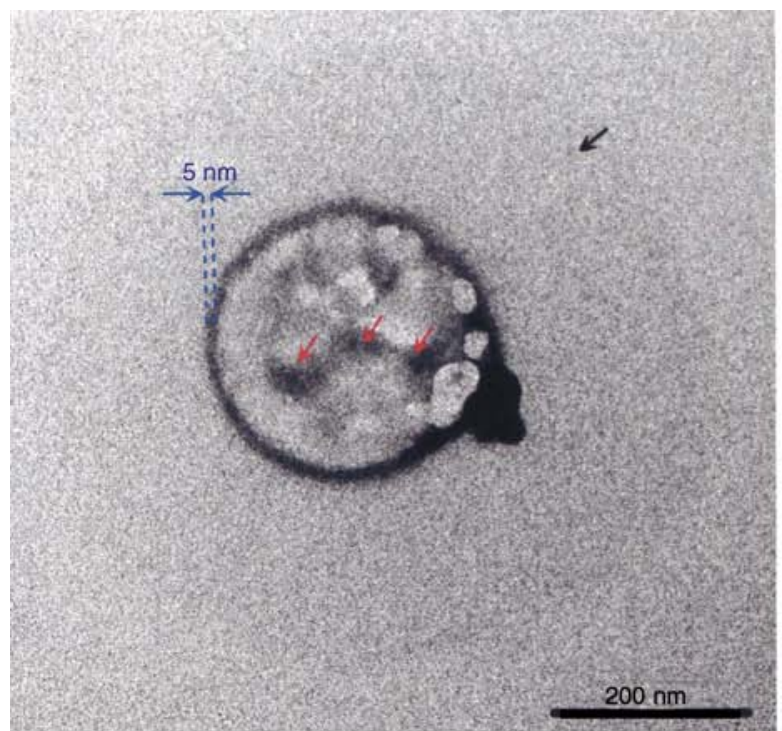

a)

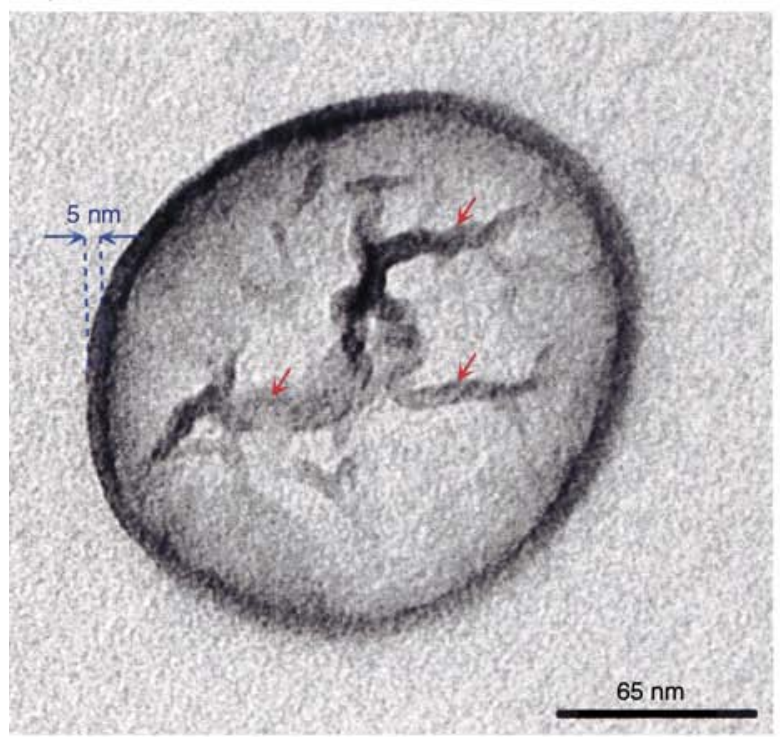

c)

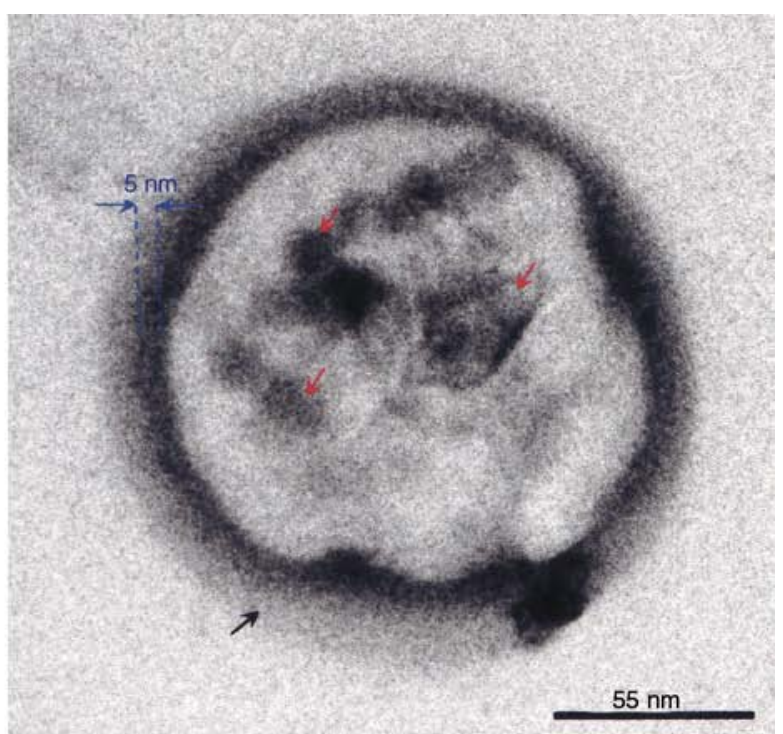

b)

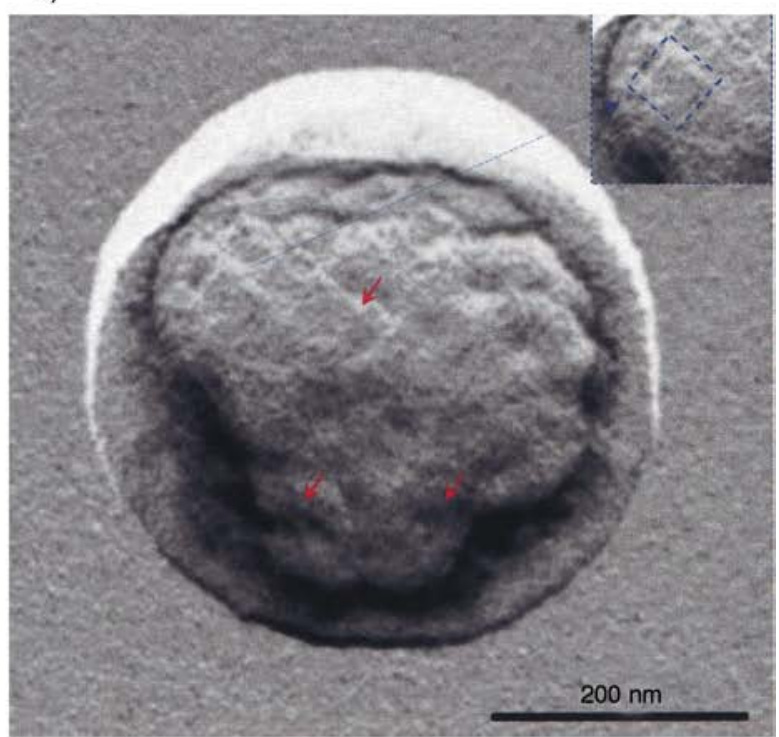

d)

Figure 12. TEM micrographs $(a, b, c)$ taken at different magnifications of poly(MePEGCA-co-HDCA) nanocapsules containing Miglyol 812. Sample (d) was shadowed with Pt/C. Red and black arrows point to pseudoregular geometries inside nanocapsules and spilled oil outside nanocapsules, respectively. Wall thickness of nanocapsules is indicated by the blue marks.

capsules and a sustained release over a period greater than $120 \mathrm{~h}$ was observed. The polymer was able to confine the oil effectively despite possible degradation of pegylated chains, and triclosan remained preferentially in the hydrophobic oil core, reducing the Burst effect and release rate.

Both nanospheres and nanocapsules showed a maximum drug release close to $80 \%$. It seems that some triclosan was entrapped in the two cases and could not diffuse effectively through the polymer matrix or polymer membrane, respectively, at an appreciable rate.
Release of triclosan from nanospheres was interpreted with the first-order equation only, since the $40 \%$ initial release could be ascribed to a Burst effect, as above indicated. The kinetic constant for this step was $0.073 \mathrm{~h}^{-1}$. In contrast, a combined model was required to interpret the release from nanocapsules. In this case, kinetic constants close to $0.145 \mathrm{~h}^{-0.5}$ and $0.024 \mathrm{~h}^{-1}$ were derived by fitting the first (Higuchi model) and second (first-order model) steps of the release. It is interesting to highlight the threefold decrease of the kinetic constant associated with the last release step, when using nanocapsules 


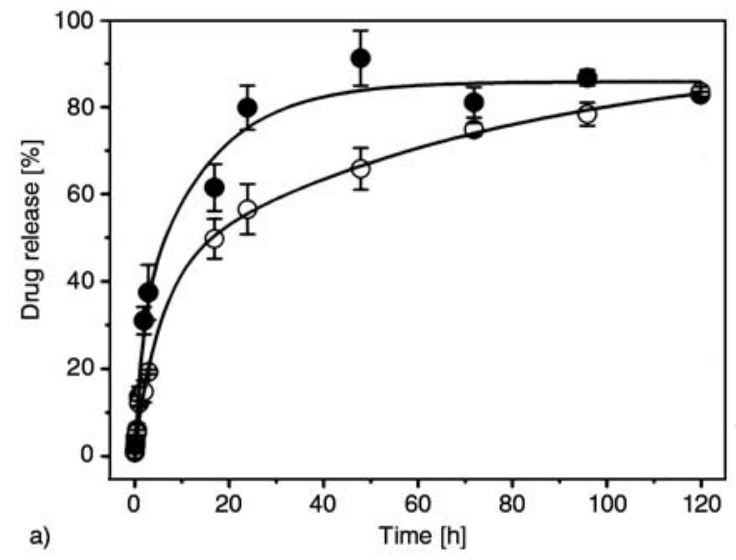

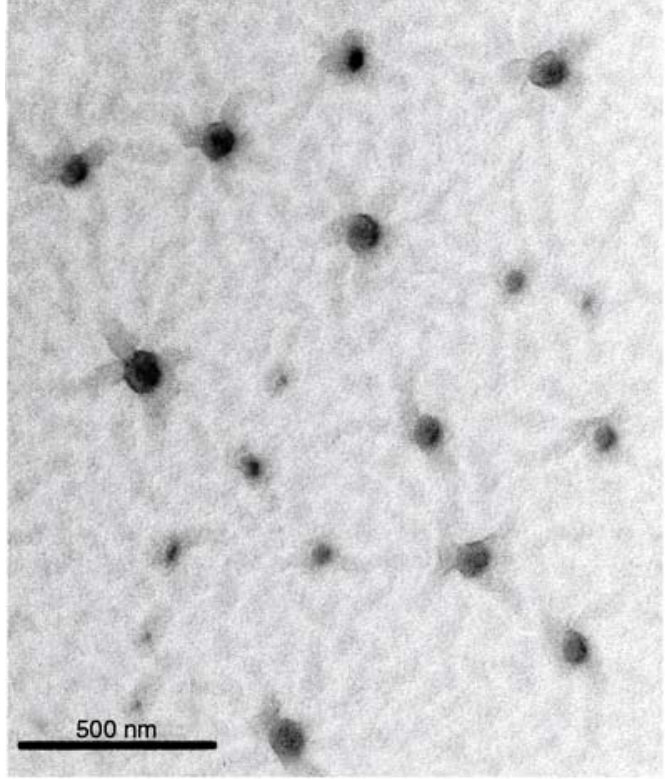

b)



c)

Figure 13. (a) Comparison between cumulative triclosan release from poly(MePEGCA-co-HDCA) nanospheres $(\bullet)$ and nanocapsules $(\mathrm{O})$ in a Sörensen/ethanol $(3 / 7 \mathrm{v} / \mathrm{v})$ medium. Vertical bars are the average values $\pm \mathrm{SD}$. Theoretical simulated curves from calculated kinetic parameters and selected release models are drawn with solid lines. (b) TEM micrograph of poly(MePEGCA-co-HDCA) nanospheres after 120 hours of exposure to the degradation medium. (c) TEM micrograph of poly(MePEGCA-co-HDCA) nanocapsules after 120 hours of exposure to the degradation medium. The blue arrow points to the decrease of the membrane thickness caused by close contact of adjacent nanocapsules.

instead of nanospheres (i.e. $0.024 \mathrm{~h}^{-1}$ versus $0.073 \mathrm{~h}^{-1}$ ). Despite the expected low diffusion rate of triclosan in the polymer matrix, the result seems logical as there is a greater affinity between triclosan and glycerol than between the drug and the polymer matrix, resulting in an enhanced release from nanospheres. It must be pointed out that the assumption of the late-time Equation (4) for both $\mathrm{NS}$ and NC dosage forms implies that the amount of drug released at each time is proportional to the residual drug inside these forms.

TEM micrographs were taken at the end of the release experiments $(120 \mathrm{~h})$ to find evidence of degradation. Nanospheres appeared clearly isolated and kept their rounded morphology and even their diameter size range (Figure 13b). Hence, no morphological changes were reported after exposure to the release medium. More interesting were the high magnification images of nanocapsules (e.g. Figure 13c) where two points merit attention: a) The membrane wall appeared well preserved and had even swelled slightly, as deduced from the increase of the wall thickness (a value close to $10 \mathrm{~nm}$ was measured). Note also that in this case the wall thickness clearly decreased upon close contact of nanocapsules. In addition, the detected degradation of pegylated chains did not affect the consistency of the nanocapsule membrane. As indicated before, 
new anionic carboxylated groups should have formed by hydrolysis, providing again a hydrophilic outer surface that stabilized the membrane in the water containing release medium; b) Drops can be clearly envisaged in the nanocapsule cores. Molecules of the release media (ethanol and water) were likely to have crossed the membrane, causing a swelling effect and microphase separation.

\subsection{Antibacterial effect of the nanospheres and nanocapsules loaded with triclosan}

The antibacterial activity of triclosan loaded nanocapsules and nanospheres was tested by direct contact with microorganisms. Figure 14a shows the relative growth of E.coli (Gram-negative bacterium) and M.luteus (Gram-positive bacterium) after 24 and 48 hours of culture in the presence of these nanospheres and nanocapsules.

E.coli was especially sensitive to the antibacterial action of triclosan. Thus, the inhibition of bacterial growth after $24 \mathrm{~h}$ of culture was about $80 \%$ for both nanospheres and nanocapsules. It may be inferred from the above results that the antibacterial effect was due to the release of approximately 50 and $80 \%$ of loaded triclosan in the nanocapsules and nanospheres, respectively (Figure 13a). The triclosan release ensured that bacterial growth inhibition was maintained around $80 \%$ after $48 \mathrm{~h}$ of culture.

M.luteus showed greater resistance to triclosan as the inhibition of bacterial growth was only about 10 and $20 \%$ after $24 \mathrm{~h}$ of culture for nanocapsules and nanospheres, respectively (Figure 14a). Notice that, as expected, inhibition was greater for the former because of their higher triclosan release rate. After $48 \mathrm{~h}$ of culture, a statistically significant increase in the inhibition of bacterial growth was reported, with values of 20 and $40 \%$ for nanocapsules and nanospheres, respectively. Note that the relative inhibition doubled with increasing the drug release period. Thus, results clearly indicate that progressive release of triclosan from both nanospheres and nanocapsules increased the antibacterial effect.

It is clear that M.luteus growth is sensitive to the amount of triclosan released, as is typical in the antimicrobial control of Gram-positive bacteria. Two approaches can be taken to solve this sensitivity problem: a) Preparation of nanospheres or nanocapsules with a higher drug load, which is not desirable since a change of the mass ratio between polymer and drug is required; b) Exposure of bacteria to a medium containing more nanospheres or nanocapsules.

Figure $14 \mathrm{~b}$ shows the antimicrobial effect of triclosan loaded nanospheres and nanocapsules in relation to the number of dosage forms, which was easily managed by controlling the dilution of the $\mathrm{NS} / \mathrm{NC}$ suspension with the broth medium. A direct linear relationship between the antibacterial effect and the number of triclosan loaded nanospheres and nanocapsules was logically found. Specifically, correlation coefficients, $r$, of 0.987 and 0.999 were obtained for the NS and NC relationships, respectively.
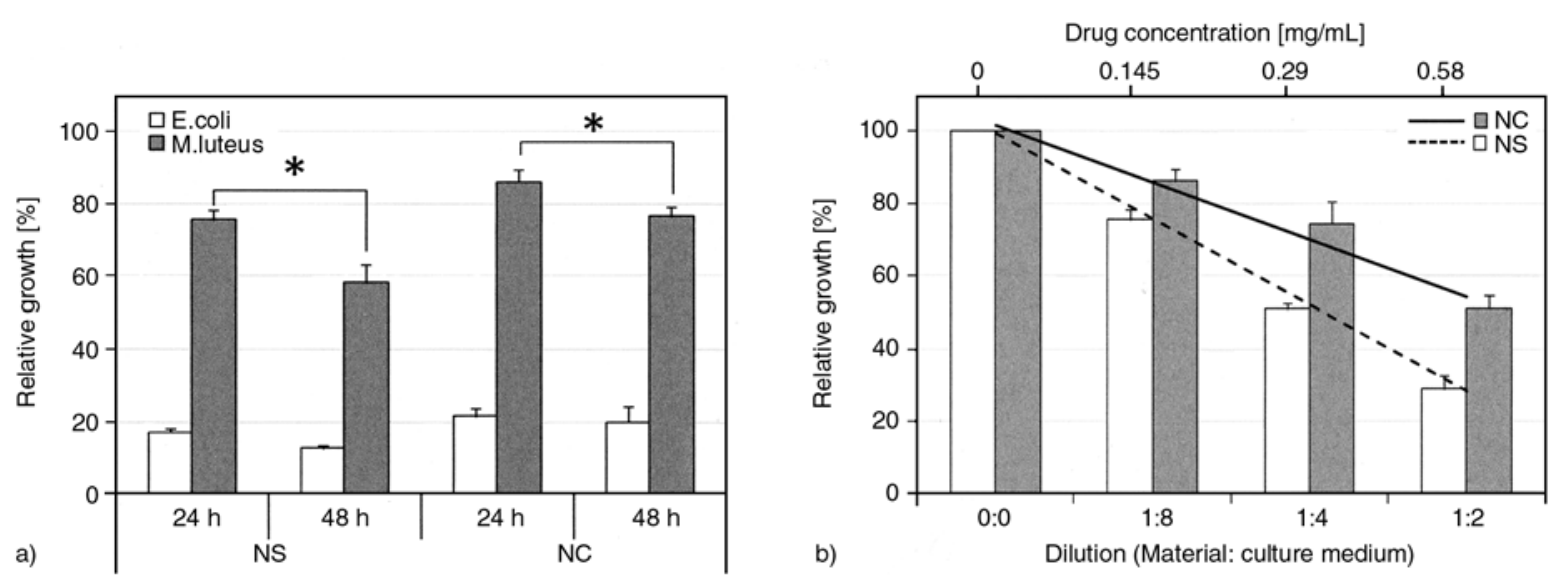

Figure 14. (a) Relative growth of E.coli and M.luteus in cultures constituted by $7 \mathrm{~mL}$ of the LC broth medium and $1 \mathrm{~mL}$ of the nanosphere (NS) or nanocapsule (NC) suspension. Asterisks indicate significantly different samples according to Tukey test, $p<0.05$. (b) Relative growth of M.luteus in cultures constituted by different dilutions of the $\mathrm{NS} / \mathrm{NC}$ suspension in the culture medium. Vertical bars are the average values $\pm \mathrm{SD}$. Solid and dashed lines indicate the relationship between relative growth and dilution for NS and NC samples. 


\section{Conclusions}

The amphiphilic copolymer derived from methylated polyethylene glycol and hexadecyl cyanoacetate esters with a hydrophilic/hydrophobic monomer ratio close to $1: 4$ was semicrystalline and able to develop large spherulites by crystallization from the melt state. Morphological observations, calorimetric data and X-ray and electron diffraction patterns pointed out the existence of two crystalline domains. These could be associated with polyethylene glycol and a hexagonal phase corresponding to the packing of the long alkyl lateral groups.

The copolymer hydrolyzed in water by cleavage of ester bonds involving the hydrophilic polyethylene glycol groups, whereas ester bonds involving the hydrophobic hexadecyl groups appeared well preserved.

The solvent displacement method coupled with the use of CIJM was highly effective in preparing triclosan loaded nanospheres and nanocapsules. Encapsulation efficiency was always close to $90 \%$ and an approximate $40 \%$ drug load was easily obtained. Morphological observations and DLS measurements indicated that NS and NC samples had size distribution centered at around 160 and $230 \mathrm{~nm}$, respectively. Nanocapsules were characterized by a polymer membrane with a thickness close to $5 \mathrm{~nm}$, a spongy structure that was easily deformed and the presence of crystallizable PEG chains in the oil core. Nanocapsules kept their morphology during exposure to the release medium (minimum $72 \mathrm{~h}$ ), although a slight swelling of the membrane wall was observed. Solvent molecules were able to cross the membrane, which was unaffected by the increase of the hydrodynamic pressure.

Nanospheres and nanocapsules exhibited a well differentiated triclosan release; specifically, the latter allowed a controlled, sustained release without Burst effect. The antimicrobial effect was also significantly different, especially when Gram-positive bacteria were assayed. A higher relative growth was always found for nanocapsules due to their lower triclosan release rate.

\section{Acknowledgements}

This research has been supported by grants from MCYT/ FEDER and AGAUR (MAT2009-11503, 2009SGR-1208). We gratefully acknowledge the important contribution of Drs. Barbara Stella and Antonello Barresi of the Università and the Politecnico di Torino (Italy), respectively, during polymer synthesis and nanoparticles preparation.

\section{References}

[1] Adams M. L., Lavasanifar A., Kwon G. S.: Amphiphilic block copolymers for drug delivery. Journal of Pharmaceutical Sciences, 92, 1343-1355 (2003).

DOI: $10.1002 /$ jps. 10397

[2] Willert M., Landfester K.: Amphiphilic copolymers from miniemulsified systems. Macromolecular Chemistry and Physics, 203, 825-836 (2002).

DOI: $10.1002 / 1521-3935(20020401) 203: 5 / 6<825:: A I D-$ MACP825>3.0.CO;2-R

[3] Storm G., Belliot S. O., Daemen T., Lasic D. D.: Surface modification of nanoparticles to oppose uptake by the mononuclear phagocyte system. Advanced Drug Delivery Reviews, 17, 31-48 (1995).

DOI: 10.1016/0169-409X(95)00039-A

[4] Gref R., Minamitake Y., Peracchia M. T., Langer R.: PEG-coated biodegradable nanospheres for intravenous administration. in 'Microparticulate systems for delivery of proteins and vaccines' (eds.: Cohen S., Bernstein H.) Marcel Dekker, New York, 279-306 (1996).

[5] Lundsted L. G., Schmolka I. R.: The synthesis and properties of block copolymer polyol surfactants. in 'Block and graft polymerization' (ed.: Ceresa R. J.) John Wiley, London 1-103 (1972).

[6] Srinivasulu B., Rao P. R., Sundaram E. V.: Synthesis and characterization of ethyl methacrylate-acrylamide copolymers. Journal of Applied Polymer Science, 43, 1521-1525 (1991).

DOI: 10.1002/app.1991.070430814

[7] Sanayei R. A., O’Driscoll K. F., Klumperman B.: Pulsed laser copolymerization of styrene and maleic anhydride. Macromolecules, 27, 5577-5582 (1994). DOI: 10.1021/ma00098a010

[8] Puig J. E., Corona-Galvan S., Maldonado A., Schultz P. C., Rodriguez B. E., Kaler E. W.: Microemulsion copolymerization of styrene and acrylic acid. Journal of Colloid and Interface Science, 137, 308-310 (1990). DOI: 10.1016/0021-9797(90)90068-Y

[9] Zhang L., Zeng Z., Chen Y., Wu C., Gao J.: Photoinitiated copolymerization of acrylamide and styrene in oil-in-water microemulsion. Journal of Applied Polymer Science, 66, 2543-2549 (1997).

DOI: $10.1002 /($ SICI)1097-4628(19971226)66:13<2543 $\because$ AID-APP17>3.0.CO;2-Y 
[10] Vašková V., Juraničová V., Bartoň J.: Polymerization in inverse microemulsions, 3. Copolymerization of water- and oil-soluble monomers initiated by radical initiators. Die Makromoleculare Chemie, 192, 13391347 (1991).

DOI: 10.1002/macp.1991.021920611

[11] Peracchia M. T., Desmaële D., Couvreur P., d'Angelo J.: Synthesis of a novel poly(MePEG cyanoacrylateco-alkyl cyanoacrylate) amphiphilic copolymer for nanoparticle technology. Macromolecules, 30, 846851 (1997).

DOI: $10.1021 / \mathrm{ma} 961453 \mathrm{k}$

[12] Peracchia M. T., Vauthier C., Desmaële D., Gulk A., Dedieu J-C., Demoy M., d'Angelo J., Couvreur P.: Pegylated nanoparticles from a novel methoxypolyethylene glycol cyanoacrylate-hexadecyl cyanoacrylate amphiphilic copolymer. Pharmaceutical Research, 15, 550-556 (1998). DOI: 10.1023/A:1011973625803

[13] Peracchia M. T., Fattal E., Desmaële D., Besnard M., Noël J. P., Gomis J. M., Appel M., d'Angelo J., Couvreur P.: Stealth ${ }^{\circledR}$ PEGylated polycyanoacrylate nanoparticles for intravenous administration and splenic targeting. Journal of Controlled Release, 60, 121-128 (1999).

DOI: 10.1016/S0168-3659(99)00063-2

[14] Brigger I., Chaminade P., Desmaële D., Peracchia M. T., d'Angelo P., Gurny R., Renoir M., Couvreur P.: Near infrared with principal component analysis as a novel analytical approach for nanoparticle technology. Pharmaceutical Research, 17, 1124-1132 (2000).

DOI: 10.1023/A:1026465931525

[15] Brambilla D., Nicolas J., Le Droumaguet B., Andrieux K., Marsaud V., Couraud P-O., Couvreur P.: Design of fluorescently tagged poly(alkyl cyanoacrylate) nanoparticles for human brain endothelial cell imaging. Chemical Communications, 46, 2602-2604 (2010). DOI: 10.1039/b924028d

[16] Le Droumaguet B., Nicolas J., Brambilla D., Mura M., Maksimenko A., de Kimpe L., Salvati E., Zona C., Airoldi C., Canovi M., Gobbi M., Magalit N., La Ferla B., Nicotra F., Scheper W., Flores O., Masserini M., Andrieux K., Couvreur P.: Versatile and efficient targeting using a single nanoparticulate platform: Application to cancer and alzheimer's disease. ACSNano, 6, 5866-5879 (2012).

DOI: $10.1021 / \mathrm{nn} 3004372$

[17] Lince F., Bolognesi S., Stella B., Marchiso D. L., Dosio F.: Preparation of polymer nanoparticles loaded with doxorubicin for controlled drug delivery. Chemical Engineering Research and Design, 89, 2410-1419 (2011).

DOI: 10.1016/j.cherd.2011.03.010
[18] Lince F., Marchisio D. L., Barresi A. A.: Smart mixers and reactors for the production of pharmaceutical nanoparticles: Proof of concept. Chemical Engineering Research and Design, 87, 543-549 (2009). DOI: $10.1016 /$ j.cherd.2008.11.009

[19] Fresta M., Cavallaro G., Giammona G., Wehrli E., Puglisi G.: Preparation and characterization of polyethyl-2-cyanoacrylate nanocapsules containing antiepileptic drugs. Biomaterials, 17, 751-758 (1996). DOI: 10.1016/0142-9612(96)81411-6

[20] Mosqueira V. C. F., Legrand P., Pinto-Alphandary H., Puisieux F., Barratt G.: Poly(D,L-lactide) nanocapsules prepared by a solvent displacement process: Influence of the composition on physicochemical and structural properties. Journal of Pharmaceutical Sciences, 89, 614-626 (2000).

DOI: 10.1002/(SICI)1520-6017(200005)89:5<614:: AID-JPS7>3.3.CO;2-Z

[21] Fessi H., Puisieux F., Devissaguet J. Ph., Ammoury N., Benita S.: Nanocapsule formation by interfacial polymer deposition following solvent displacement. International Journal of Pharmaceutics, 55, R1-R4 (1989). DOI: $10.1016 / 0378-5173(89) 90281-0$

[22] Gibaldi M., Feldman S.: Establishment of sink conditions in dissolution rate determinations. Theoretical considerations and application to nondisintegrating dosage forms. Journal of Pharmaceutical Sciences, 56, 1238-1242 (1967).

DOI: $10.1002 / j p s .2600561005$

[23] Wagner J. G.: Interpretation of percent dissolved-time plots derived from in vitro testing of conventional tablets and capsules. Journal of Pharmaceutical Sciences, 58, 1253-1257 (1969). DOI: $10.1002 / j p s .2600581021$

[24] Higuchi T.: Rate of release of medicaments from ointment bases containing drugs in suspension. Journal of Pharmaceutical Sciences, 50, 874-879 (1961).

DOI: $\underline{10.1002 / j p s .2600501018}$

[25] Higuchi T.: Mechanism of sustained-action medication. Theoretical analysis of rate of release of solid drugs dispersed in solid matrices. Journal of Pharmaceutical Sciences, 52, 1145-1149 (1963).

DOI: $10.1002 / j p s .2600521210$

[26] Baker R. W.: Controlled release of biologically active agents. Wiley, New York (1987).

[27] Lestel L., Guegan P., Boileau S., Cheradame H., Laupretre F.: Influence of the chemical nature of crosslinks on the local dynamics of bulk poly(ethylene oxide) networks as studied by carbon-13 NMR at temperatures well above the glass-transition temperature. Macromolecules, 25, 6024-6028 (1992).

DOI: $10.1021 / \mathrm{ma} 00048 \mathrm{a} 025$ 
[28] Muthukumar M., Ober C. K., Thomas E. L.: Competing interactions and levels of ordering in self-organizing polymeric materials. Science, 277, 1225-1232 (1998). DOI: $10.1126 /$ science. 277.5330 .1225

[29] Zhu L., Chen Y., Zhang A., Calhoun B. H., Chun M., Quirk R. P., Cheng S. Z. D., Hsiao B. S., Yeh F., Hashimoto T.: Phase structures and morphologies determined by competitions among self-organization, crystallization, and vitrification in a disordered poly (ethylene oxide)- $b$-polystyrene diblock copolymer. Physical Reviews B, 60, 10022-10031 (1999). DOI: 10.1103/PhysRevB.60.10022

[30] Ryan A. J., Hamley I. W., Bras W., Bates F. S.: Structure development in semicrystalline diblock copolymers crystallizing from the ordered melt. Macromolecules, 28, 3860-3868 (1995).

DOI: $10.1021 / \mathrm{ma} 00115 \mathrm{a} 016$

[31] Schäffer E., Thurn-Albrecht T. P., Russell T. P., Steiner U.: Electrically induced structure formation and pattern transfer. Nature, 403, 874-877 (2000). DOI: $10.1038 / 35002540$

[32] Kawai T., Rahman N., Matsuba G., Nishida K., Kanaya T., Nakano M., Okamoto H., Kawada J., Usuki A., Honma N., Nakajima K., Matsuda M.: Crystallization and melting behavior of poly (L-lactic acid). Macromolecules, 40, 9463-9469 (2007). DOI: $10.1021 / \mathrm{ma} 070082 \mathrm{c}$

[33] Müller A. J., Arnal M. L., Balsamo V.: Crystallization in block copolymers with more than one crystallizable block. in 'Lecture notes in physics: Progress in understanding of polymer crystallization' (eds.: Reiter G., Strobl G.) Springer, Berlin, Vol 714, 229-259 (2007). DOI: $10.1007 / 3-540-47307-613$
[34] Nandan B., Hsu J-Y., Chen H-L.: Crystallization behavior of crystalline-amorphous diblock copolymers consisting of a rubbery amorphous block. Polymer Reviews, 46, 143-172 (2006). DOI: $10.1080 / 15321790600646802$

[35] Nielsen J. R., Hathaway C. E.: Infrared spectra of normal paraffins in the hexagonal crystal form. Journal Molecular Spectroscopy, 10, 366-377 (1963).

DOI: 10.1016/0022-2852(63)90182-6

[36] Takahashi Y., Tadokoro H.: Structural studies of polyethers, $\left(-\left(\mathrm{CH}_{2}\right)_{\mathrm{m}}-\mathrm{O}-\right)_{\mathrm{n}}$. X. Crystal structure of poly (ethylene oxide)). Macromolecules, 6, 672-675 (1973). DOI: $10.1021 / \mathrm{ma} 60035 \mathrm{a} 005$

[37] Lotz B., Kovacs A. J.: Properties of copolymers composed of one poly-ethylene-oxide and one polystyrene block. I. Preparation, composition and microscopy study of single crystal (in French). Colloid and Polymer Science, 209, 97-114 (1966).

DOI: $10.1007 / \mathrm{BF} 01500628$

[38] Lotz B., Kovacs A. J., Bassett G. A., Keller A.: Properties of copolymers composed of one poly-ethyleneoxide and one polystyrene block II. Morphology of single crystals. Colloid and Polymer Science, 209, 115-124 (1966).

DOI: $10.1007 / \mathrm{BF} 01500629$

[39] Panyam J., Labhasetwar V.: Biodegradable nanoparticles for drug and gene delivery to cells and tissue. Advanced Drug Delivery Reviews, 55, 329-347 (2003). DOI: 10.1016/S0169-409X(02)00228-4

[40] Zurita R., Puiggalí J., Rodríguez-Galán A.: Triclosan release from coated polyglycolide threads. Macromolecular Bioscience, 6, 58-69 (2006).

DOI: $\underline{10.1002 / \mathrm{mabi} .200500147}$ 